\title{
EVOLUTIONARY VARIATIONAL INEQUALITIES ARISING IN QUASISTATIC FRICTIONAL CONTACT PROBLEMS FOR ELASTIC MATERIALS
}

\author{
DUMITRU MOTREANU AND MIRCEA SOFONEA
}

Received 28 December 1999

We consider a class of evolutionary variational inequalities arising in quasistatic frictional contact problems for linear elastic materials. We indicate sufficient conditions in order to have the existence, the uniqueness and the Lipschitz continuous dependence of the solution with respect to the data, respectively. The existence of the solution is obtained using a time-discretization method, compactness and lower semicontinuity arguments. In the study of the discrete problems we use a recent result obtained by the authors (2000). Further, we apply the abstract results in the study of a number of mechanical problems modeling the frictional contact between a deformable body and a foundation. The material is assumed to have linear elastic behavior and the processes are quasistatic. The first problem concerns a model with normal compliance and a version of Coulomb's law of dry friction, for which we prove the existence of a weak solution. We then consider a problem of bilateral contact with Tresca's friction law and a problem involving a simplified version of Coulomb's friction law. For these two problems we prove the existence, the uniqueness and the Lipschitz continuous dependence of the weak solution with respect to the data.

\section{Introduction}

This work concerns the study of a class of abstract evolutionary variational inequalities modeling the frictional contact between an elastic body and a foundation. Situations which involve dynamic or quasistatic frictional contact abound in industry, especially in engines, motors and transmissions. For this reason there exists a considerable engineering literature dealing with frictional contact problems. An early attempt to study frictional contact problems within the framework of variational inequalities was made in [6]. An excellent reference on analysis and numerical approximations of contact problems involving elastic materials with or without friction is [8]. The mathematical, mechanical and numerical state of the art can be found in [14]. 
Quasistatic contact problems arise when the forces applied to a system vary slowly in time so that acceleration is negligible. The mathematical treatment of quasistatic problems is recent. The reason lies in the considerable difficulties that the process of frictional contact presents in the modeling and the analysis because of the complicated surface phenomena involved. The variational analysis of some quasistatic contact problems can be found for instance in $[2,3,5,9]$ within linearized elasticity.

In a variational form, a number of quasistatic frictional contact problems for linear elastic materials lead to variational models of the form: find a displacement field $u$ : $[0, T] \rightarrow V$ such that

$$
\begin{gathered}
a(u(t), v-\dot{u}(t))+j(u(t), v)-j(u(t), \dot{u}(t)) \geq(f(t), v-\dot{u}(t))_{V} \quad \forall v \in V, \text { a.e. } t \in(0, T), \\
u(0)=u_{0} .
\end{gathered}
$$

Here, $V$ is a function space of admissible displacements, $a$ is a bilinear form related to the elastic coefficients and the functional $j$ is determined by the type of contact and friction boundary conditions. The data $f$ is related to the given body forces and surface tractions, and $u_{0}$ represents the initial displacement. In (1.1) and everywhere in this paper, $T>0,[0, T]$ is the time interval of interest, and the dot above a quantity denotes the derivative of the quantity with respect to the time variable $t$.

Abstract evolutionary inequalities of the form (1.1) and (1.2) were considered in [4], in the case when $j$ does not depend on the solution, that is, $j(u, v)=j(v)$ for all $v \in V$. There, the existence and uniqueness of the solution was proved using arguments of the theory of nonlinear evolution equations with maximal monotone operators in a real Hilbert space. Considering the case when the nondifferentiable functional $j$ depends on the solution of the problem leads to a new and nonstandard mathematical problem.

The aim of this paper is to provide variational analysis for abstract Cauchy problems of the form (1.1) and (1.2) and to apply these results in the study of some quasistatic elastic frictional contact problems. Thus, we provide sufficiency conditions on the nondifferentiable functional $j$ in order to have the existence, the uniqueness and the Lipschitz continuous dependence of the solution with respect to the data, respectively. Some of these conditions are formulated in terms of the directional derivative of $j$ which consists, to the best of our knowledge, a trait of novelty of this paper. The proof of the existence result for (1.1) and (1.2) is based on a time-discretization method, similar to that used in $[1,5]$ in the study of quasistatic contact problems for elastic or viscoplastic materials. Given a time step, we construct a sequence of quasivariational inequalities for which we prove the existence of the solution using a result recently obtained in [11]. Then, we interpolate the discrete solution in time and, using compactness and lower semicontinuity arguments, we derive the existence of a solution to (1.1) and (1.2). The uniqueness of the solution as well as its Lipschitz continuous dependence with respect to the data is proved under additional assumptions on the functional $j$, by using standard Gronwall-type arguments.

Next, we consider a problem of frictional contact between an elastic body and a foundation. We assume that the body forces and surface tractions acting upon the body vary slowly in time so that the acceleration in the system is negligible. Neglecting the acceleration term in the equation of motion leads to a quasistatic approach of the process. 
We model the contact with a general normal compliance condition, similar to the one in $[7,15]$. In this condition the interpenetration of the body's surface into the foundation is allowed and may be justified by considering the interpenetration and deformation of surface asperities. The friction is modeled with a quasistatic version of Coulomb's law. We prove that the mechanical problem leads to a variational formulation of the form (1.1) and (1.2) in which $u$ represents the displacement field. Then, using the abstract results obtained in the study of the Cauchy problem (1.1) and (1.2), we establish the existence of a weak solution of the model, under a smallness assumption concerning the contact and frictional boundary conditions. This result completes the results obtained in $[2,3,9]$ where quasistatic contact problems with normal compliance and friction involving linear elastic materials were considered. We also present a quasistatic elastic problem modeling the bilateral contact with Tresca's friction law as well as a quasistatic contact problem with a simplified version of Coulomb's law. For both these problems we prove the existence, the uniqueness and the Lipschitz continuous dependence of the solution with respect to the data and therefore we extend some results presented in $[6,13]$, where the corresponding static problems are considered.

The paper is structured as follows. In Section 2, we introduce the notation, list the assumptions on the data and state our main result, Theorem 2.1. The proof of this result is carried out in several steps in Section 3. In Section 4, we describe the elastic problem with normal compliance and friction, set it into a variational formulation and state an existence result, Theorem 4.1. The proof of Theorem 4.1 is given in Section 5 and it is based on the abstract result provided by Theorem 2.1. Finally, in Section 6 we study the model of bilateral contact with Tresca's friction law as well as the model involving a simplified version of Coulomb's friction law.

\section{The abstract problem}

Let $V$ be a real Hilbert space endowed with the inner product $(\cdot, \cdot)_{V}$ and the associated norm $|\cdot|_{V}$. We denote by " $\longrightarrow$ " and " $\rightarrow$ " the weak convergence and the strong convergence on $V$, respectively. In what follows $0_{V}$ will represent the zero element of $V$. For $p \in[1, \infty]$, we use the standard notation for $L^{p}(0, T ; V)$ spaces. We also use the Sobolev space $W^{1, \infty}(0, T ; V)$ with the norm

$$
|u|_{W^{1, \infty}(0, T ; V)}=|u|_{L^{\infty}(0, T ; V)}+|\dot{u}|_{L^{\infty}(0, T ; V)},
$$

where a dot now represents the weak derivative with respect to the time variable.

In the study of (1.1) and (1.2) we consider the following assumptions.

(1) $a: V \times V \rightarrow \mathbb{R}$ is a bilinear symmetric form and

(a) there exists $M>0$ such that $|a(u, v)| \leq M|u|_{V}|v|_{V}$ for all $u, v \in V$;

(b) there exists $m>0$ such that $a(v, v) \geq m|v|_{V}^{2}$ for all $v \in V$.

(2) $j: V \times V \rightarrow \mathbb{R}$ and for every $\eta \in V, j(\eta, \cdot): V \rightarrow \mathbb{R}$ is a positively homogeneous subadditive functional, that is,

(a) $j(\eta, \lambda u)=\lambda j(\eta, u)$ for all $u \in V, \lambda \in \mathbb{R}_{+}$;

(b) $j(\eta, u+v) \leq j(\eta, u)+j(\eta, v)$ for all $u, v \in V$. 
Evolutionary variational inequalities arising in quasistatic ...

$$
\begin{gathered}
f \in W^{1, \infty}(0, T ; V), \\
u_{0} \in V, \\
a\left(u_{0}, v\right)+j\left(u_{0}, v\right) \geq(f(0), v)_{V} \quad \forall v \in V .
\end{gathered}
$$

Keeping in mind (2), it results that for all $\eta \in V, j(\eta, \cdot): V \rightarrow \mathbb{R}$ is a convex functional. Therefore, there exists the directional derivative $j_{2}^{\prime}$ given by

$$
j_{2}^{\prime}(\eta, u ; v)=\lim _{\lambda \rightarrow 0^{+}} \frac{1}{\lambda}[j(\eta, u+\lambda v)-j(\eta, u)] \quad \forall \eta, u, v \in V .
$$

We consider now the following additional assumptions on the functional $j$.

(j1) For every sequence $\left\{u_{n}\right\} \subset V$ with $\left|u_{n}\right|_{V} \rightarrow \infty$, every sequence $\left\{t_{n}\right\} \subset[0,1]$ and each $\bar{u} \in V$ one has

$$
\liminf _{n \rightarrow \infty}\left[\frac{1}{\left|u_{n}\right|_{V}^{2}} j_{2}^{\prime}\left(t_{n} u_{n}, u_{n}-\bar{u} ;-u_{n}\right)\right]<m .
$$

(j2) For every sequence $\left\{u_{n}\right\} \subset V$ with $\left|u_{n}\right|_{V} \rightarrow \infty$, every bounded sequence $\left\{\eta_{n}\right\} \subset V$ and each $\bar{u} \in V$, one has

$$
\liminf _{n \rightarrow \infty}\left[\frac{1}{\left|u_{n}\right|_{V}^{2}} j_{2}^{\prime}\left(\eta_{n}, u_{n}-\bar{u} ;-u_{n}\right)\right]<m .
$$

(j3) For all sequences $\left\{u_{n}\right\} \subset V$ and $\left\{\eta_{n}\right\} \subset V$ such that $u_{n} \rightarrow u \in V, \eta_{n} \rightarrow \eta \in V$ and for every $v \in V$, the inequality below holds

$$
\limsup _{n \rightarrow \infty}\left[j\left(\eta_{n}, v\right)-j\left(\eta_{n}, u_{n}\right)\right] \leq j(\eta, v)-j(\eta, u) .
$$

(j4) There exists $c_{0} \in(0, m)$ such that

$$
j(u, v-u)-j(v, v-u) \leq c_{0}|u-v|_{V}^{2} \quad \forall u, v \in V .
$$

(j5) There exist two functions $a_{1}: V \rightarrow \mathbb{R}$ and $a_{2}: V \rightarrow \mathbb{R}$ which map bounded sets in $V$ into bounded sets in $\mathbb{R}$ such that $|j(\eta, u)| \leq a_{1}(\eta)|u|_{V}^{2}+a_{2}(\eta) \forall \eta, u \in V$, and $a_{1}\left(0_{V}\right)<m-c_{0}$.

(j6) For every sequence $\left\{\eta_{n}\right\} \subset V$ with $\eta_{n} \rightarrow \eta \in V$, and every bounded sequence $\left\{u_{n}\right\} \subset V$, one has

$$
\lim _{n \rightarrow \infty}\left[j\left(\eta_{n}, u_{n}\right)-j\left(\eta, u_{n}\right)\right]=0 .
$$

(j7) For every $s \in(0, T]$ and every functions $u, v \in W^{1, \infty}(0, T ; V)$ with $u(0)=$ $v(0), u(s) \neq v(s)$, the inequality below holds

$$
\int_{0}^{s}[j(u(t), \dot{v}(t))-j(u(t), \dot{u}(t))+j(v(t), \dot{u}(t))-j(v(t), \dot{v}(t))] d t<\frac{m}{2}|u(s)-v(s)|_{V}^{2} .
$$


(j8) There exists $\alpha \in(0, m / 2)$ such that for every $s \in(0, T]$ and every functions $u, v \in W^{1, \infty}(0, T ; V)$ with $u(s) \neq v(s)$, the inequality below holds

$$
\int_{0}^{s}[j(u(t), \dot{v}(t))-j(u(t), \dot{u}(t))+j(v(t), \dot{u}(t))-j(v(t), \dot{v}(t))] d t<\alpha|u(s)-v(s)|_{V}^{2} \text {. }
$$

Our main result, which we establish in the next section is the following.

Theorem 2.1. Let (1), (2), (2.2), (2.3), and (2.4) hold. Then:

$\left(1^{\prime}\right)$ Under the assumptions $(j 1)-(j 6)$ there exists at least a solution $u \in W^{1, \infty}(0, T ; V)$ to the problem (1.1) and (1.2).

$\left(2^{\prime}\right)$ Under the assumptions $(j 1)-(j 7)$ there exists a unique solution $u \in W^{1, \infty}(0, T ; V)$ to the problem (1.1) and (1.2).

$\left(3^{\prime}\right)$ Under the assumptions $(j 1)-(j 6)$ and (j8) there exists a unique solution $u=$ $u\left(f, u_{0}\right) \in W^{1, \infty}(0, T ; V)$ to the problem (1.1) and (1.2) and the mapping $\left(f, u_{0}\right) \mapsto u$ is Lipschitz continuous from $W^{1, \infty}(0, T ; V) \times V$ to $L^{\infty}(0, T ; V)$.

The proof of Theorem 2.1 will be established in the next section. Here we remark that if $\varphi: V \rightarrow \mathbb{R}_{+}$is a continuous seminorm then the functional $j$ defined by $j(u, v)=\varphi(v)$ for all $u, v \in V$ satisfies the assumptions (2), (j1)-(j8). Therefore, from Theorem 2.1 we deduce the following result, which represents a version of Proposition II.9 in [4].

COROllaRy 2.2. Let (1), (2.2), (2.3) hold, let $\varphi: V \rightarrow \mathbb{R}_{+}$be a continuous seminorm and let us suppose that $u_{0}$ satisfies the condition

$$
a\left(u_{0}, v\right)+\varphi(v) \geq(f(0), v)_{V} \quad \forall v \in V .
$$

Then, there exists a unique function $u \in W^{1, \infty}(0, T ; V)$ such that

$$
\begin{gathered}
a(u(t), v-\dot{u}(t))+\varphi(v)-\varphi(\dot{u}(t)) \geq(f(t), v-\dot{u}(t))_{V} \quad \forall v \in V, \text { a.e. } t \in(0, T), \\
u(0)=u_{0} .
\end{gathered}
$$

Moreover, the mapping $\left(f, u_{0}\right) \mapsto u$ is Lipschitz continuous from $W^{1, \infty}(0, T ; V) \times V$ to $L^{\infty}(0, T ; V)$.

\section{Proof of Theorem 2.1}

The proof of Theorem 2.1 will be carried out in several steps, using a time-discretization method, compactness and lower semicontinuity arguments. The first step is based on a result obtained recently in [11] that we recall here in a simplified version, for the convenience of the reader.

Theorem 3.1. Let (1), (2), (j1)-(j3) hold. Then, for all $f \in V$ there exists at least an element $u \in V$ such that

$$
a(u, v-u)+j(u, v)-j(u, u) \geq(f, v-u)_{V} \quad \forall v \in V .
$$


Evolutionary variational inequalities arising in quasistatic ...

The proof of Theorem 3.1 is based on standard arguments of elliptic variational inequalities, topological degree theory and fixed point. A trait of novelty of Theorem 3.1 consists, to the best of our knowledge, in considering conditions formulated in terms of the directional derivative of the functional $j$, in the study of quasivariational inequalities of the form (3.1).

We turn now to the proof of Theorem 2.1. To this end we suppose in what follows that (1), (2), (2.2), (2.3), (2.4) hold and the assumptions (j1)-(j6) are fulfilled. Let $n \in \mathbb{N}$. We consider the following implicit scheme: find $u_{n}^{i+1} \in V$ such that

$$
\begin{aligned}
a\left(u_{n}^{i+1}, v-\frac{n}{T}\left(u_{n}^{i+1}-u_{n}^{i}\right)\right) & +j\left(u_{n}^{i+1}, v\right)-j\left(u_{n}^{i+1}, \frac{n}{T}\left(u_{n}^{i+1}-u_{n}^{i}\right)\right) \\
\geq & \left(f\left(\frac{T(i+1)}{n}\right), v-\frac{n}{T}\left(u_{n}^{i+1}-u_{n}^{i}\right)\right)_{V} \quad \forall v \in V,
\end{aligned}
$$

where $u_{n}^{0}=u_{0}, i=0,1, \ldots, n-1$.

In the first step we prove the solvability of the quasivariational inequality (3.2) and we provide estimates of the solution to this problem.

Lemma 3.2. There exists at least a solution $u_{n}^{i+1}$ to the quasivariational inequality (3.2), for $i=0,1, \ldots, n-1$. Moreover, the solution satisfies

$$
\begin{gathered}
\left|u_{n}^{i+1}\right|_{V}^{2} \leq \frac{1}{m-c_{0}-a_{1}\left(0_{V}\right)}\left(\left|f\left(\frac{T(i+1)}{n}\right)\right|_{V}\left|u_{n}^{i+1}\right|_{V}+a_{2}\left(0_{V}\right)\right), \\
\left|u_{n}^{i+1}-u_{n}^{i}\right|_{V} \leq \frac{1}{m-c_{0}}\left|f\left(\frac{T(i+1)}{n}\right)-f\left(\frac{T i}{n}\right)\right|_{V}
\end{gathered}
$$

for all $i=0,1, \ldots, n-1$.

Proof. Let $i \in\{0,1, \ldots, n-1\}$. Using (2)(a) and setting $w=(T / n) v+u_{n}^{i}$ it follows that (3.2) is equivalent to the inequality

$$
\begin{aligned}
& a\left(u_{n}^{i+1}, w-u_{n}^{i+1}\right)+j\left(u_{n}^{i+1},\right.\left.w-u_{n}^{i}\right)-j\left(u_{n}^{i+1}, u_{n}^{i+1}-u_{n}^{i}\right) \\
& \geq\left(f\left(\frac{T(i+1)}{n}\right), w-u_{n}^{i+1}\right)_{V} \quad \forall w \in V .
\end{aligned}
$$

Now using Theorem 3.1, we obtain the existence of the solution to (3.5) and the equivalence of problems (3.2) and (3.5) yields the existence part of the lemma.

Taking now $w=0_{V}$ in (3.5) and using (2)(b), we find

$$
\begin{aligned}
a\left(u_{n}^{i+1}, u_{n}^{i+1}\right) & \leq\left(f\left(\frac{T(i+1)}{n}\right), u_{n}^{i+1}\right)_{V}+j\left(u_{n}^{i+1},-u_{n}^{i}\right)-j\left(u_{n}^{i+1}, u_{n}^{i+1}-u_{n}^{i}\right) \\
& \leq\left|f\left(\frac{T(i+1)}{n}\right)\right|_{V}\left|u_{n}^{i+1}\right|_{V}+j\left(u_{n}^{i+1},-u_{n}^{i+1}\right),
\end{aligned}
$$

and (1)(b) yields

$$
m\left|u_{n}^{i+1}\right|_{V}^{2} \leq\left|f\left(\frac{T(i+1)}{n}\right)\right|_{V}\left|u_{n}^{i+1}\right|_{V}+j\left(u_{n}^{i+1},-u_{n}^{i+1}\right) .
$$


Taking $u=u_{n}^{i+1}$ and $v=0_{V}$ in (j4) and using (j5) with $\eta=0_{V}$, we obtain

$$
j\left(u_{n}^{i+1},-u_{n}^{i+1}\right) \leq c_{0}\left|u_{n}^{i+1}\right|_{V}^{2}+j\left(0_{V},-u_{n}^{i+1}\right) \leq\left(c_{0}+a_{1}\left(0_{V}\right)\right)\left|u_{n}^{i+1}\right|_{V}^{2}+a_{2}\left(0_{V}\right) \text {. }
$$

Since $a_{1}\left(0_{V}\right)<m-c_{0}$, the estimate (3.3) results from (3.7) and (3.8).

Using again (2)(a) it follows that $j\left(u, 0_{V}\right)=\lambda j\left(u, 0_{V}\right)$ for all $u \in V$ and $\lambda>0$, which implies

$$
j\left(u, 0_{V}\right)=0 \quad \forall u \in V .
$$

Setting $w=u_{n}^{i}$ in (3.5) and using (3.9), it follows that

$$
\begin{aligned}
& a\left(u_{n}^{i+1}, u_{n}^{i+1}-u_{n}^{i}\right) \\
& \quad \leq\left(f\left(\frac{T(i+1)}{n}\right), u_{n}^{i+1}-u_{n}^{i}\right)_{V}-j\left(u_{n}^{i+1}, u_{n}^{i+1}-u_{n}^{i}\right) \quad \forall i=0,1, \ldots, n-1 .
\end{aligned}
$$

Using again (3.5) with $i-1$ in place of $i$ and $w=u_{n}^{i+1}$, we find

$$
\begin{aligned}
& a\left(u_{n}^{i}, u_{n}^{i+1}-u_{n}^{i}\right)+ j\left(u_{n}^{i}, u_{n}^{i+1}-u_{n}^{i-1}\right)-j\left(u_{n}^{i}, u_{n}^{i}-u_{n}^{i-1}\right) \\
& \geq\left(f\left(\frac{T i}{n}\right), u_{n}^{i+1}-u_{n}^{i}\right)_{V} \quad \forall i=1, \ldots, n-1
\end{aligned}
$$

and, keeping in mind (2)(b), (2.4), we obtain

$$
\begin{aligned}
& -a\left(u_{n}^{i}, u_{n}^{i+1}-u_{n}^{i}\right) \\
& \quad \leq\left(-f\left(\frac{T i}{n}\right), u_{n}^{i+1}-u_{n}^{i}\right)_{V}+j\left(u_{n}^{i}, u_{n}^{i+1}-u_{n}^{i}\right) \quad \forall i=0,1, \ldots, n-1 .
\end{aligned}
$$

It follows now from (1)(b), (3.10), (3.12), and $(\mathrm{j} 4)$ that

$$
\begin{aligned}
& m\left|u_{n}^{i+1}-u_{n}^{i}\right|_{V}^{2} \leq a\left(u_{n}^{i+1}-u_{n}^{i}, u_{n}^{i+1}-u_{n}^{i}\right) \\
& \quad \leq\left(f\left(\frac{T(i+1)}{n}\right)-f\left(\frac{T i}{n}\right), u_{n}^{i+1}-u_{n}^{i}\right)_{V}-j\left(u_{n}^{i+1}, u_{n}^{i+1}-u_{n}^{i}\right)+j\left(u_{n}^{i}, u_{n}^{i+1}-u_{n}^{i}\right) \\
& \quad \leq\left|f\left(\frac{T(i+1)}{n}\right)-f\left(\frac{T i}{n}\right)\right|_{V}\left|u_{n}^{i+1}-u_{n}^{i}\right|_{V}+c_{0}\left|u_{n}^{i+1}-u_{n}^{i}\right|_{V}^{2} \quad \forall i=0,1, \ldots, n-1,
\end{aligned}
$$

which implies (3.4).

We now consider the functions $u_{n}:[0, T] \rightarrow V$ and $\tilde{u}_{n}:[0, T] \rightarrow V$ defined as follows:

$$
\begin{gathered}
u_{n}(0)=u_{0}, \quad u_{n}(t)=u_{n}^{i}+\frac{n t-T i}{T}\left(u_{n}^{i+1}-u_{n}^{i}\right) \quad \forall t \in\left(\frac{T i}{n}, \frac{T(i+1)}{n}\right], \\
\tilde{u}_{n}(0)=u_{0}, \quad \tilde{u}_{n}(t)=u_{n}^{i+1} \quad \forall t \in\left(\frac{T i}{n}, \frac{T(i+1)}{n}\right]
\end{gathered}
$$

where $u_{n}^{0}=u_{0}, u_{n}^{i+1}$ solves (3.2) and $i=0,1, \ldots, n-1$. 
262 Evolutionary variational inequalities arising in quasistatic ...

In the next step we provide convergence results involving the sequences $\left\{u_{n}\right\}$ and $\left\{\tilde{u}_{n}\right\}$.

Lemмa 3.3. There exist an element $u \in W^{1, \infty}(0, T ; V)$ and subsequences of the sequences $\left\{u_{n}\right\}$ and $\left\{\tilde{u}_{n}\right\}$, again denoted $\left\{u_{n}\right\}$ and $\left\{\tilde{u}_{n}\right\}$, respectively, such that

$$
\begin{aligned}
& u_{n} \longrightarrow u \text { weakly* in } L^{\infty}(0, T ; V) \text {, } \\
& \dot{u}_{n} \longrightarrow \dot{u} \text { weakly* in } L^{\infty}(0, T ; V) \text {, } \\
& \tilde{u}_{n}(t) \longrightarrow u(t) \text { weakly in } V \text {, a.e. } t \in(0, T) \text {. }
\end{aligned}
$$

Proof. Let $n \in \mathbb{N}$. Using (3.14) it follows that $u_{n}:[0, T] \rightarrow V$ is an absolutely continuous function and its derivative is given by

$$
\dot{u}_{n}(t)=\frac{n}{T}\left(u_{n}^{i+1}-u_{n}^{i}\right) \quad \text { a.e. } t \in\left(\frac{T i}{n}, \frac{T(i+1)}{n}\right), i=0,1, \ldots, n-1 .
$$

Therefore, from (3.3), (3.4), (3.14), and (3.19), we deduce

$$
\begin{aligned}
\left|u_{n}(t)\right|_{V} \leq & \left|u_{0}\right|_{V}+\frac{1}{m-c_{0}}\left|f\left(\frac{T}{n}\right)-f(0)\right|_{V} \text { a.e. } t \in\left(0, \frac{T}{n}\right), \\
\left|u_{n}(t)\right|_{V} \leq & \frac{1}{\left(m-c_{0}-a_{1}\left(0_{V}\right)\right)^{1 / 2}}\left(\left|f\left(\frac{T i}{n}\right)\right|_{V}\left|u_{n}^{i}\right|_{V}+a_{2}\left(0_{V}\right)\right)^{1 / 2} \\
& +\frac{1}{m-c_{0}}\left|f\left(\frac{T(i+1)}{n}\right)-f\left(\frac{T i}{n}\right)\right|_{V} \\
\text { a.e. } t \in\left(\frac{T i}{n}, \frac{T(i+1)}{n}\right), i=1, \ldots, n-1, & \text { a.e. } t \in\left(\frac{T i}{n}, \frac{T(i+1)}{n}\right), i=0,1, \ldots, n-1 .
\end{aligned}
$$

Keeping in mind the regularity (2.2) and estimate (3.3), from the previous inequalities it follows that $u_{n} \in W^{1, \infty}(0, T ; V)$ and

$$
\left|u_{n}\right|_{W^{1, \infty}(0, T ; V)} \leq C .
$$

Here and everywhere in this section $C$ represents a positive constant which may depend on $f$ and $u_{0}$ but does not depend on $n$ and whose value may change from place to place.

The existence of an element $u \in W^{1, \infty}(0, T ; V)$ as well as the convergences (3.16) and (3.17) follow from standard compactness arguments.

We turn now to the proof of (3.18). To this end we remark that the convergence results (3.16) and (3.17) imply

$$
u_{n}(t) \longrightarrow u(t) \quad \text { weakly in } V, \quad \forall t \in[0, T] .
$$


Moreover, using again (3.14), (3.15), and (3.4) we find

$$
\begin{aligned}
\left|u_{n}(t)-\tilde{u}_{n}(t)\right|_{V}= & \left(1-\frac{n t-T i}{T}\right)\left|u_{n}^{i+1}-u_{n}^{i}\right|_{V} \\
\leq & \frac{1}{m-c_{0}}\left|f\left(\frac{T(i+1)}{n}\right)-f\left(\frac{T i}{n}\right)\right|_{V} \\
& \forall t \in\left(\frac{T i}{n}, \frac{T(i+1)}{n}\right], i=0,1, \ldots, n-1
\end{aligned}
$$

and, keeping in mind the regularity (2.2), we deduce

$$
\left|u_{n}-\tilde{u}_{n}\right|_{L^{\infty}(0, T ; V)} \leq \frac{1}{m-c_{0}} \cdot \frac{T}{n}|\dot{f}|_{L^{\infty}(0, T ; V)} .
$$

This inequality proves that

$$
u_{n}-\tilde{u}_{n} \longrightarrow 0 \quad \text { in } L^{\infty}(0, T ; V)
$$

and therefore

$$
u_{n}(t)-\tilde{u}_{n}(t) \longrightarrow 0 \text { a.e. } t \in(0, T) .
$$

The convergence (3.18) is now a consequence of (3.22) and (3.26).

In the next two steps we prove additional convergence and semicontinuity results. To this end, for every $n \in \mathbb{N}$ consider the function $f_{n}:[0, T] \rightarrow V$ defined as follows:

$$
f_{n}(0)=f(0), \quad f_{n}(t)=f\left(\frac{T(i+1)}{n}\right) \quad \forall t \in\left(\frac{T i}{n}, \frac{T(i+1)}{n}\right], i=0,1, \ldots, n-1 .
$$

Everywhere in what follows $u$ will denote the element of $W^{1, \infty}(0, T ; V)$ whose existence was proved in Lemma 3.3 and $\left\{u_{n}\right\},\left\{\tilde{u}_{n}\right\},\left\{f_{n}\right\}$ will represent appropriate subsequences of the sequences $\left\{u_{n}\right\},\left\{\tilde{u}_{n}\right\}$, and $\left\{f_{n}\right\}$, respectively.

Lemma 3.4. The following properties hold:

$$
\begin{gathered}
\lim _{n \rightarrow \infty} \int_{0}^{T} a\left(\tilde{u}_{n}(t), g(t)\right) d t=\int_{0}^{T} a(u(t), g(t)) d t \quad \forall g \in L^{2}(0, T ; V), \\
\liminf _{n \rightarrow \infty} \int_{0}^{T} a\left(\tilde{u}_{n}(t), \dot{u}_{n}(t)\right) d t \geq \int_{0}^{T} a(u(t), \dot{u}(t)) d t, \\
\lim _{n \rightarrow \infty} \int_{0}^{T}\left(f_{n}(t), g(t)-\dot{u}_{n}(t)\right)_{V} d t \\
=\int_{0}^{T}(f(t), g(t)-\dot{u}(t))_{V} d t \quad \forall g \in L^{2}(0, T ; V) .
\end{gathered}
$$

Proof. It follows from (3.16) and (3.25) that $\tilde{u}_{n} \rightarrow u$ weakly in $L^{2}(0, T ; V)$ and therefore, keeping in mind (1), we deduce (3.28). Using again (1)(a), (3.25), and (3.21) 
264 Evolutionary variational inequalities arising in quasistatic ...

we find

$$
\lim _{n \rightarrow \infty} \int_{0}^{T} a\left(\tilde{u}_{n}(t)-u_{n}(t), \dot{u}_{n}(t)\right) d t=0
$$

and, from (3.22), $u_{n}(0)=u_{0}$ and standard semicontinuity arguments, we obtain

$$
\liminf _{n \rightarrow \infty} \int_{0}^{T} a\left(u_{n}(t), \dot{u}_{n}(t)\right) d t \geq \int_{0}^{T} a(u(t), \dot{u}(t)) d t .
$$

The inequality (3.29) is now a consequence of (3.31) and (3.32).

Finally, from (2.2) and (3.27) we obtain that the sequence $\left\{f_{n}\right\}$ converges uniformly to $f$ on $[0, T]$, that is,

$$
\max _{t \in[0, T]}\left|f_{n}(t)-f(t)\right|_{V} \longrightarrow 0 \quad \forall t \in[0, T] .
$$

The convergence (3.30) is now a consequence of (3.17) and (3.33).

LEMMA 3.5. The following properties hold:

$$
\begin{array}{rl}
\limsup _{n \rightarrow \infty} \int_{0}^{T} & j\left(\tilde{u}_{n}(t), g(t)\right) d t \leq \int_{0}^{T} j(u(t), g(t)) d t \quad \forall g \in L^{2}(0, T ; V), \\
& \limsup _{n \rightarrow \infty} \int_{0}^{T}\left[j\left(u(t), \dot{u}_{n}(t)\right)-j\left(\tilde{u}_{n}(t), \dot{u}_{n}(t)\right)\right] d t \leq 0, \\
& \liminf _{n \rightarrow \infty} \int_{0}^{T} j\left(u(t), \dot{u}_{n}(t)\right) d t \geq \int_{0}^{T} j(u(t), \dot{u}(t)) d t .
\end{array}
$$

Proof. Let $g \in L^{2}(0, T ; V)$. Using (2.2), (3.3), and (3.15), it follows that $\left\{\tilde{u}_{n}(t)\right\}$ is a bounded sequence in $V$, for all $t \in[0, T]$. Therefore, by assumption (j5) we deduce that there exists $C>0$ such that

$$
\left|j\left(\tilde{u}_{n}(t), g(t)\right)\right| \leq C\left(|g(t)|_{V}^{2}+1\right) \quad \text { a.e. } t \in(0, T), \forall n \in \mathbb{N} .
$$

This inequality allows us to apply Fatou's lemma to obtain

$$
\limsup _{n \rightarrow \infty} \int_{0}^{T} j\left(\tilde{u}_{n}(t), g(t)\right) d t \leq \int_{0}^{T} \limsup _{n \rightarrow \infty} j\left(\tilde{u}_{n}(t), g(t)\right) d t .
$$

We apply (3.18) and assumption ( j6) to find

$$
\lim _{n \rightarrow \infty} j\left(\tilde{u}_{n}(t), g(t)\right)=j(u(t), g(t)) \quad \text { a.e. } t \in(0, T) .
$$

The inequality (3.34) is now a consequence of (3.38) and (3.39).

Now, using again assumption (j5) and (3.21) we deduce that there exists $C>0$ such that

$$
\left|j\left(u(t), \dot{u}_{n}(t)\right)-j\left(\tilde{u}_{n}(t), \dot{u}_{n}(t)\right)\right| \leq C \quad \text { a.e. } t \in(0, T), \forall n \in \mathbb{N} \text {. }
$$


This inequality allows us to apply again Fatou's lemma to obtain

$$
\begin{aligned}
& \limsup _{n \rightarrow \infty} \int_{0}^{T}[j(u(t)\left.\left., \dot{u}_{n}(t)\right)-j\left(\tilde{u}_{n}(t), \dot{u}_{n}(t)\right)\right] d t \\
& \leq \int_{0}^{T} \limsup _{n \rightarrow \infty}\left[j\left(u(t), \dot{u}_{n}(t)\right)-j\left(\tilde{u}_{n}(t), \dot{u}_{n}(t)\right)\right] d t .
\end{aligned}
$$

Moreover, using (3.18), (3.21), and assumption (j6), we deduce

$$
\lim _{n \rightarrow \infty}\left[j\left(u(t), \dot{u}_{n}(t)\right)-j\left(\tilde{u}_{n}(t), \dot{u}_{n}(t)\right)\right]=0 \quad \text { a.e. } t \in(0, T) .
$$

Inequality (3.35) follows now from (3.41) and (3.42).

Finally, inequality (3.36) follows from standard semicontinuity arguments, keeping in mind (2), (j5), and (3.17).

We have now all the ingredients to prove the theorem.

Proof of Theorem 2.1. (1) Using (3.2), (3.15), (3.19), and (3.27) we obtain

$$
\begin{aligned}
a\left(\tilde{u}_{n}(t), v-\dot{u}_{n}(t)\right)_{V}+ & j\left(\tilde{u}_{n}(t), v\right)-j\left(\tilde{u}_{n}(t), \dot{u}_{n}(t)\right) \\
& \geq\left(f_{n}(t), v-\dot{u}_{n}(t)\right)_{V} \quad \forall v \in V, \text { a.e. } t \in(0, T) .
\end{aligned}
$$

This inequality and assumption ( $\mathrm{j} 5)$ yield

$$
\begin{gathered}
\int_{0}^{T} a\left(\tilde{u}_{n}(t), g(t)-\dot{u}_{n}(t)\right) d t+\int_{0}^{T} j\left(\tilde{u}_{n}(t), g(t)\right) d t-\int_{0}^{T} j\left(\tilde{u}_{n}(t), \dot{u}_{n}(t)\right) d t \\
\geq \int_{0}^{T}\left(f_{n}(t), g(t)-\dot{u}_{n}(t)_{V}\right) d t \quad \forall g \in L^{2}(0, T ; V) .
\end{gathered}
$$

Now using (3.28), (3.29), (3.30), (3.34), (3.35), (3.36), and (3.44) we find

$$
\begin{gathered}
\int_{0}^{T} a(u(t), g(t)-\dot{u}(t)) d t+\int_{0}^{T} j(u(t), g(t)) d t-\int_{0}^{T} j(u(t), \dot{u}(t)) d t \\
\geq \int_{0}^{T}(f(t), g(t)-\dot{u}(t))_{V} d t \quad \forall g \in L^{2}(0, T ; V) .
\end{gathered}
$$

Using now in (3.45) a classical application of Lebesgue point for $L^{1}$ functions we obtain that $u \in W^{1, \infty}(0, T ; V)$ satisfies (1.1) and from (3.14) and (3.22) we deduce (1.2) which concludes the proof.

(2) Consider two solutions $u_{1}, u_{2} \in W^{1, \infty}(0, T ; V)$ to the Cauchy problem (1.1) and (1.2). The inequalities below hold for all $v \in V$ and a.e. $t \in(0, T)$ :

$$
\begin{aligned}
& a\left(u_{1}(t), v-\dot{u}_{1}(t)\right)+j\left(u_{1}(t), v\right)-j\left(u_{1}(t), \dot{u}_{1}(t)\right) \geq\left(f(t), v-\dot{u}_{1}(t)\right)_{V}, \\
& a\left(u_{2}(t), v-\dot{u}_{2}(t)\right)+j\left(u_{2}(t), v\right)-j\left(u_{2}(t), \dot{u}_{2}(t)\right) \geq\left(f(t), v-\dot{u}_{2}(t)\right)_{V} .
\end{aligned}
$$


266 Evolutionary variational inequalities arising in quasistatic ...

We take $v=\dot{u}_{2}(t)$ in the first inequality, $v=\dot{u}_{1}(t)$ in the second inequality. Adding the corresponding inequalities and using (1) we obtain

$$
\begin{aligned}
& \frac{1}{2} \frac{d}{d t} a\left(u_{1}(t)-u_{2}(t), u_{1}(t)-u_{2}(t)\right) \\
& \quad \leq j\left(u_{1}(t), \dot{u}_{2}(t)\right)-j\left(u_{1}(t), \dot{u}_{1}(t)\right)+j\left(u_{2}(t), \dot{u}_{1}(t)\right)-j\left(u_{2}(t), \dot{u}_{2}(t)\right) \text { a.e. } t \in(0, T) .
\end{aligned}
$$

Moreover, from (1.2) we have

$$
u_{1}(0)=u_{2}(0)=u_{0}
$$

Arguing by contradiction, let us suppose that $u_{1} \neq u_{2}$. Then there exists $s \in(0, T]$ such that

$$
u_{1}(s) \neq u_{2}(s) .
$$

Integrating (3.47) over $[0, s]$, by using (1)(b) and (3.48) yields

$$
\begin{aligned}
& \frac{m}{2}\left|u_{1}(s)-u_{2}(s)\right|_{V}^{2} \\
& \quad \leq \int_{0}^{s}\left[j\left(u_{1}(t), \dot{u}_{2}(t)\right)-j\left(u_{1}(t), \dot{u}_{1}(t)\right)+j\left(u_{2}(t), \dot{u}_{1}(t)\right)-j\left(u_{2}(t), \dot{u}_{2}(t)\right)\right] d t .
\end{aligned}
$$

In view of (3.48), (3.49) and assumption (j7), the inequality (3.50) leads to a contradiction, which concludes the proof.

(3) The unique solvability of the Cauchy problem (1.1) and (1.2) follows from (2) since the assumption (j8) implies (j7). Let now $f_{i} \in W^{1, \infty}(0, T ; V)$ and $u_{0 i} \in V$ be such that (2.4) holds for $i=1,2$. We denote in what follows by $u_{i} \in W^{1, \infty}(0, T ; V)$ the solution of the Cauchy problem (1.1) and (1.2) for the data $f_{i}$ and $u_{0 i}$. A computation similar to the one in (3.47) leads to the inequality

$$
\begin{aligned}
\frac{1}{2} \frac{d}{d t} a & \left(u_{1}(t)-u_{2}(t), u_{1}(t)-u_{2}(t)\right) \\
\leq & j\left(u_{1}(t), \dot{u}_{2}(t)\right)-j\left(u_{1}(t), \dot{u}_{1}(t)\right)+j\left(u_{2}(t), \dot{u}_{1}(t)\right) \\
& \quad-j\left(u_{2}(t), \dot{u}_{2}(t)\right)+\left(f_{1}(t)-f_{2}(t), \dot{u}_{1}(t)-\dot{u}_{2}(t)\right)_{V} \quad \text { a.e. } t \in(0, T) .
\end{aligned}
$$

We suppose in what follows that $u_{1} \neq u_{2}$ and let $s \in(0, T]$ be such that $u_{1}(s) \neq u_{2}(s)$. Integrating over $[0, s]$ the previous inequality, using the initial conditions $u_{i}(0)=u_{0 i}$ and (1), yields

$$
\begin{aligned}
\frac{m}{2}\left|u_{1}(s)-u_{2}(s)\right|_{V}^{2} \leq & \frac{M}{2}\left|u_{01}-u_{02}\right|_{V}^{2}+\int_{0}^{s}\left[j\left(u_{1}(t), \dot{u}_{2}(t)\right)-j\left(u_{1}(t), \dot{u}_{1}(t)\right)\right. \\
& \left.+j\left(u_{2}(t), \dot{u}_{1}(t)\right)-j\left(u_{2}(t), \dot{u}_{2}(t)\right)\right] d t \\
& +\int_{0}^{s}\left(f_{1}(t)-f_{2}(t), \dot{u}_{1}(t)-\dot{u}_{2}(t)\right)_{V} d t
\end{aligned}
$$


In view of the assumption ( j8) we obtain

$$
\left(\frac{m}{2}-\alpha\right)\left|u_{1}(s)-u_{2}(s)\right|_{V}^{2} \leq \frac{M}{2}\left|u_{01}-u_{02}\right|_{V}^{2}+\int_{0}^{s}\left(f_{1}(t)-f_{2}(t), \dot{u}_{1}(t)-\dot{u}_{2}(t)\right)_{V} d t .
$$

Let $\delta \in(0, m-2 \alpha)$. Using the inequality

$$
a b \leq \frac{a^{2}}{2 \delta}+\frac{\delta b^{2}}{2}
$$

we obtain

$$
\begin{aligned}
& \int_{0}^{s}\left(f_{1}(t)-f_{2}(t), \dot{u}_{1}(t)-\dot{u}_{2}(t)\right)_{V} d t \\
&=\left(f_{1}(s)-f_{2}(s), u_{1}(s)-u_{2}(s)\right)_{V}-\left(f_{1}(0)-f_{2}(0), u_{01}-u_{01}\right)_{V} \\
&-\int_{0}^{s}\left(\dot{f}_{1}(t)-\dot{f}_{2}(t), u_{1}(t)-u_{2}(t)\right)_{V} d t \\
& \leq \frac{1}{2 \delta}\left|f_{1}(s)-f_{2}(s)\right|_{V}^{2}+\frac{\delta}{2}\left|u_{1}(s)-u_{2}(s)\right|_{V}^{2}+\frac{1}{2 \delta}\left|f_{1}(0)-f_{2}(0)\right|_{V}^{2} \\
&+\frac{\delta}{2}\left|u_{01}-u_{02}\right|_{V}^{2}+\frac{1}{2 \delta} \int_{0}^{s}\left|\dot{f}_{1}(t)-\dot{f}_{2}(t)\right|_{V}^{2} d t+\frac{\delta}{2} \int_{0}^{s}\left|u_{1}(t)-u_{2}(t)\right|_{V}^{2} d t \\
& \leq \frac{T+2}{2 \delta}\left|f_{1}-f_{2}\right|_{W^{1, \infty}(0, T ; V)}^{2}+\frac{\delta}{2}\left|u_{1}(s)-u_{2}(s)\right|_{V}^{2} \\
&+\frac{\delta}{2}\left|u_{01}-u_{02}\right|_{V}^{2}+\frac{\delta}{2} \int_{0}^{s}\left|u_{1}(t)-u_{2}(t)\right|_{V}^{2} d t
\end{aligned}
$$

Keeping in mind (3.53) and the previous inequality we deduce

$$
\begin{aligned}
\left|u_{1}(s)-u_{2}(s)\right|_{V}^{2} d t \leq & C_{1}\left(\left|u_{01}-u_{02}\right|_{V}^{2}+\left|f_{1}-f_{2}\right|_{W^{1, \infty}(0, T ; V)}^{2}\right) \\
& +C_{2} \int_{0}^{s}\left|u_{1}(t)-u_{2}(t)\right|_{V}^{2} d t,
\end{aligned}
$$

where $C_{1}, C_{2}>0$ depend on $M, m, \alpha, \delta$, and $T$. Clearly the inequality (3.56) holds for all $s \in[0, T]$. Using now a Gronwall-type argument, from (3.56) we obtain

$$
\left|u_{1}(0)-u_{2}(1)\right|_{V}^{2} d t \leq C\left(\left|u_{01}-u_{02}\right|_{V}^{2}+\left|f_{1}-f_{2}\right|_{W^{1, \infty}(0, T ; V)}^{2}\right) \quad \forall s \in[0, T],
$$

where $C>0$, which concludes the proof.

\section{A frictional contact problem with normal compliance}

In this section, we present an application of Theorem 2.1 in the study of a nonlinear problem modeling the contact between an elastic body and a foundation.

The physical setting is as follows. A linear elastic body occupies a bounded domain $\Omega \subset \mathbb{R}^{d}(d=2,3)$ with a regular boundary $\Gamma$ that is partitioned into three disjoint 
measurable parts $\Gamma_{1}, \Gamma_{2}$, and $\Gamma_{3}$, such that meas $\left(\Gamma_{1}\right)>0$. Let $T>0$ and let $[0, T]$ denote the time interval of interest. The body is clamped on $\Gamma_{1} \times(0, T)$ and thus the displacement field vanishes there. A volume force of density $f_{0}$ acts in $\Omega \times(0, T)$ and a surface traction of density $f_{2}$ acts on $\Gamma_{2} \times(0, T)$. We assume that the forces and tractions change slowly in time so that the acceleration in the system is negligible. The boundary conditions on the potential contact surface $\Gamma_{3}$ involve normal compliance and friction and will be discussed below.

Under these conditions, the classical formulation of the mechanical problem of frictional contact of the elastic body is the following.

Problem 1. Find a displacement $\boldsymbol{u}: \Omega \times[0, T] \rightarrow \mathbb{R}^{d}$ and a stress field $\boldsymbol{\sigma}: \Omega \times$ $[0, T] \rightarrow S_{d}$ such that

$$
\begin{aligned}
& \boldsymbol{\sigma}=\mathscr{E} \boldsymbol{\varepsilon}(\boldsymbol{u}) \quad \text { in } \Omega \times(0, T) \text {, } \\
& \operatorname{Div} \boldsymbol{\sigma}+\boldsymbol{f}_{0}=\mathbf{0} \text { in } \Omega \times(0, T) \text {, } \\
& \boldsymbol{u}=\mathbf{0} \text { on } \Gamma_{1} \times(0, T), \\
& \boldsymbol{\sigma} \boldsymbol{v}=\boldsymbol{f}_{2} \quad \text { on } \Gamma_{2} \times(0, T) \text {, } \\
& -\sigma_{v}=p_{v}\left(u_{v}-g\right) \text {, } \\
& \left|\sigma_{\tau}\right| \leq p_{\tau}\left(u_{v}-g\right) \\
& \left.\begin{array}{l}
\left|\boldsymbol{\sigma}_{\tau}\right|<p_{\tau}\left(u_{v}-g\right) \Longrightarrow \dot{\boldsymbol{u}}_{\tau}=0 \\
\left|\boldsymbol{\sigma}_{\tau}\right|=p_{\tau}\left(u_{v}-g\right) \Longrightarrow \boldsymbol{\sigma}_{\tau}=-\lambda \dot{\boldsymbol{u}}_{\tau}, \quad \lambda \geq 0
\end{array}\right\} \text { on } \Gamma_{3} \times(0, T), \\
& \boldsymbol{u}(0)=\boldsymbol{u}_{0} \quad \text { in } \Omega \text {. }
\end{aligned}
$$

Here $S_{d}$ represents the space of second order symmetric tensors on $\mathbb{R}^{d}$. Relation (4.1) is the elastic constitutive law in which $\mathscr{E}$ is a fourth order tensor and $\boldsymbol{\varepsilon}(\boldsymbol{u})$ is the infinitesimal strain tensor. Relation (4.2) represents the equilibrium equation, equations (4.3) and (4.4) are the displacement-traction boundary conditions in which $\boldsymbol{v}$ represents the unit outward normal vector to $\Gamma$ and, finally, the function $\boldsymbol{u}_{0}$ in (4.7) denotes the initial displacement.

We make some comments on the contact conditions (4.5) and (4.6) in which $\sigma_{v}$ denotes the normal stress, $\sigma_{\tau}$ represents the tangential traction, $u_{v}$ is the normal displacement and $\dot{\boldsymbol{u}}_{\tau}$ represents the tangential velocity. The equality (4.5) represents the normal compliance contact condition in which $p_{\nu}$ is a prescribed nonnegative function and $g$ denotes the gap between the potential contact surface $\Gamma_{3}$ and the foundation, measured along the direction of the outward normal $v$. When positive, $u_{v}-g$ represents the penetration of the surface asperities into those of the foundation. Such contact condition was proposed in [10] and used in a number of publications, see, for example, $[2,3,9,15,16]$ and references there. In this condition the interpenetration is allowed but penalized. An example of a normal compliance function $p_{v}$ is

$$
p_{v}(r)=c_{\nu} r_{+}
$$


where $c_{v}$ is a positive constant and $r_{+}=\max \{0, r\}$. Formally, Signorini's nonpenetration condition is obtained in the limit $c_{v} \rightarrow \infty$.

The relations (4.6) represent a version of Coulomb's law of dry friction in which $p_{\tau}$ is a prescribed nonnegative function, the so-called friction bound. According to (4.6) the tangential shear cannot exceed the maximal frictional resistance $p_{\tau}\left(u_{v}-g\right)$. Then, if the strict inequality holds, the surface adheres to the foundation and is in the so-called stick state, and when equality holds there is relative sliding, the so-called slip state. Therefore, at each time instant the potential contact surface $\Gamma_{3}$ is divided into three zones: the stick zone, the slip zone and the zone of separation, in which $u_{v}<g$ and there is no contact. The boundaries of these zones are unknown a priori and form free boundaries. The choice

$$
p_{\tau}=\mu p_{\nu},
$$

leads to the usual Coulomb's law, and $\mu \geq 0$ is the coefficient of friction (cf. [6] or [13]). Recently a modified version of the Coulomb friction law was derived in [17, 18] from thermodynamic considerations. It consists of using the friction law (4.6) with

$$
p_{\tau}=\mu p_{\nu}\left(1-\delta p_{\nu}\right)_{+},
$$

where $\delta$ is a small positive material constant related to the wear and hardness of the surface. Contact and frictional boundary conditions of the form (4.5) and (4.6) were considered in [15] in the study of quasistatic process for Kelvin-Voigt viscoelastic materials.

To provide the variational analysis of the problem (4.1), (4.2), (4.3), (4.4), (4.5), (4.6), and (4.7) we introduce the following notation. We define the inner products and the corresponding norms on $\mathbb{R}^{d}$ and $S_{d}$ by

$$
\begin{array}{cl}
\boldsymbol{u} \cdot \boldsymbol{v}=u_{i} v_{i}, & |\boldsymbol{v}|=(\boldsymbol{v} \cdot \boldsymbol{v})^{1 / 2}, \quad \forall \boldsymbol{u}, \boldsymbol{v} \in \mathbb{R}^{d}, \\
\boldsymbol{\sigma} \cdot \boldsymbol{\tau}=\sigma_{i j} \tau_{i j}, & |\boldsymbol{\tau}|=(\boldsymbol{\tau} \cdot \boldsymbol{\tau})^{1 / 2}, \quad \forall \boldsymbol{\sigma}, \boldsymbol{\tau} \in S_{d} .
\end{array}
$$

Here and below the indices $i$ and $j$ run between 1 and $d$, the summation convention over repeated indices is used, and the index following a comma indicates a partial derivative. Next, we use the following spaces:

$$
\begin{gathered}
H=\left\{\boldsymbol{v}=\left(v_{i}\right) \mid v_{i} \in L^{2}(\Omega)\right\}=L^{2}(\Omega)^{d}, \\
H_{1}=\left\{\boldsymbol{v}=\left(v_{i}\right) \mid v_{i} \in H^{1}(\Omega)\right\}=H^{1}(\Omega)^{d}, \\
\mathscr{H}=\left\{\boldsymbol{\tau}=\left(\tau_{i j}\right) \mid \tau_{i j}=\tau_{j i} \in L^{2}(\Omega)\right\}=L^{2}(\Omega)_{s}^{d \times d}, \\
\mathscr{H}_{1}=\left\{\boldsymbol{\tau} \in \mathscr{H} \mid \tau_{i j, j} \in H\right\} .
\end{gathered}
$$

These are real Hilbert spaces endowed with the inner products

$$
\begin{aligned}
(\boldsymbol{u}, \boldsymbol{v})_{H}=\int_{\Omega} u_{i} v_{i} d x, & (\boldsymbol{\sigma}, \boldsymbol{\tau})_{\mathscr{H}}=\int_{\Omega} \sigma_{i j} \tau_{i j} d x, \\
(\boldsymbol{u}, \boldsymbol{v})_{H_{1}}=(\boldsymbol{u}, \boldsymbol{v})_{H}+(\boldsymbol{\varepsilon}(\boldsymbol{u}), \boldsymbol{\varepsilon}(\boldsymbol{v}))_{\mathscr{H}}, & (\boldsymbol{\sigma}, \boldsymbol{\tau})_{\mathscr{H}_{1}}=(\boldsymbol{\sigma}, \boldsymbol{\tau})_{\mathscr{H}}+(\operatorname{Div} \boldsymbol{\sigma}, \operatorname{Div} \boldsymbol{\tau})_{H},
\end{aligned}
$$


with the associated norms $|\cdot|_{H},|\cdot| \mathscr{H},|\cdot|_{H_{1}}$, and $|\cdot| \mathscr{H}_{1}$, respectively. Here $\boldsymbol{\varepsilon}: H_{1} \rightarrow \mathscr{H}$ and Div : $\mathscr{H}_{1} \rightarrow H$ are the deformation and divergence operators, respectively, defined by

$$
\boldsymbol{\varepsilon}(\boldsymbol{u})=\left(\varepsilon_{i j}(\boldsymbol{u})\right), \quad \varepsilon_{i j}(\boldsymbol{u})=\frac{1}{2}\left(u_{i, j}+u_{j, i}\right), \quad \operatorname{Div} \boldsymbol{\sigma}=\left(\sigma_{i j, j}\right) .
$$

For an element $\boldsymbol{v} \in H_{1}$ we denote by $\boldsymbol{v}$ its trace on $\Gamma$ and by $v_{\nu}=\boldsymbol{v} \cdot \boldsymbol{v}$ and $\boldsymbol{v}_{\tau}=\boldsymbol{v}-v_{\nu} \boldsymbol{v}$ its normal and tangential components on the boundary. Let $V$ be the closed subspace of $H_{1}$ given by

$$
V=\left\{\boldsymbol{v} \in H_{1} \mid \boldsymbol{v}=\mathbf{0} \text { on } \Gamma_{1}\right\} .
$$

Since meas $\left(\Gamma_{1}\right)>0$, the following Korn's inequality holds:

$$
|\boldsymbol{\varepsilon}(\boldsymbol{v})|_{\mathscr{H}} \geq c_{K}|\boldsymbol{v}|_{H_{1}} \quad \forall \boldsymbol{v} \in V,
$$

where $c_{K}>0$ is a constant depending only on $\Omega$ and $\Gamma_{1}$. A proof of Korn's inequality can be found in, for instance, [12, page 79]. Over the space $V$ we consider the inner product given by

$$
(\boldsymbol{u}, \boldsymbol{v})_{V}=(\boldsymbol{\varepsilon}(\boldsymbol{u}), \boldsymbol{\varepsilon}(\boldsymbol{v}))_{\mathscr{H}}
$$

and let $|\cdot|_{V}$ be the associated norm. It follows from Korn's inequality (4.16) that $|\cdot|_{H_{1}}$ and $|\cdot|_{V}$ are equivalent norms on $V$. Therefore $\left(V,|\cdot|_{V}\right)$ is a real Hilbert space. Moreover, by the Sobolev trace theorem and (4.16) and (4.17), we have a constant $c_{B}$ depending only on the domain $\Omega, \Gamma_{1}$ and $\Gamma_{3}$ such that

$$
|\boldsymbol{v}|_{L^{2}\left(\Gamma_{3}\right)^{d}} \leq c_{B}|\boldsymbol{v}|_{V} \quad \forall v \in V .
$$

In the study of the mechanical problem (4.1), (4.2), (4.3), (4.4), (4.5), (4.6), and (4.7) we assume that $\mathscr{E}: \Omega \times S_{d} \rightarrow S_{d}$ is a bounded symmetric positive definite fourth order tensor, that is,

(i) (a) $\mathscr{E}_{i j k l} \in L^{\infty}(\Omega), 1 \leq i, j, k, l \leq d$;

(b) $\mathscr{E} \sigma \cdot \tau=\sigma \cdot \mathscr{E} \tau$, for all $\sigma, \tau \in S_{d}$, a.e. in $\Omega$;

(c) there exists $m>0$ such that $\mathscr{E} \boldsymbol{\tau} \cdot \boldsymbol{\tau} \geq m|\boldsymbol{\tau}|^{2}$ for all $\boldsymbol{\tau} \in S_{d}$, a.e. in $\Omega$.

The functions $p_{r}: \Gamma_{3} \times \mathbb{R} \rightarrow \mathbb{R}_{+}(r=v, \tau)$ satisfy:

(ii) (a) there exists $L_{r}>0$ such that $\left|p_{r}\left(\boldsymbol{x}, u_{1}\right)-p_{r}\left(\boldsymbol{x}, u_{2}\right)\right| \leq L_{r}\left|u_{1}-u_{2}\right|$ for all $u_{1}, u_{2} \in \mathbb{R}$, a.e. $\boldsymbol{x} \in \Gamma_{3}$;

(b) $\boldsymbol{x} \mapsto p_{r}(\boldsymbol{x}, u)$ is Lebesgue measurable on $\Gamma_{3}$ for all $u \in \mathbb{R}$;

(c) $\boldsymbol{x} \mapsto p_{r}(\boldsymbol{x}, u)=0$ for $u \leq 0$, a.e. $\boldsymbol{x} \in \Gamma_{3}$.

The assumptions (ii) on $p_{v}$ and $p_{\tau}$ are fairly general. The main restriction is the requirement that, asymptotically, the functions grow at most linearly. Clearly, the function defined in (4.8) satisfies this condition. We also observe that if the functions $p_{\nu}$ and $p_{\tau}$ are related by (4.9) or (4.10) and $p_{v}$ satisfies condition (ii)(a), then $p_{\tau}$ does too with $L_{\tau}=\mu L_{\nu}$.

The forces and tractions are assumed to satisfy

$$
\boldsymbol{f}_{0} \in W^{1, \infty}(0, T ; H), \quad \boldsymbol{f}_{2} \in W^{1, \infty}\left(0, T ; L^{2}\left(\Gamma_{2}\right)^{d}\right),
$$


and the gap function satisfies

$$
g \in L^{2}\left(\Gamma_{3}\right), \quad g \geq 0 \text { a.e. on } \Gamma_{3} .
$$

Next we define the bilinear form $a: V \times V \rightarrow \mathbb{R}$ by

$$
a(\boldsymbol{u}, \boldsymbol{v})=(\mathscr{E} \boldsymbol{\varepsilon}(\boldsymbol{u}), \boldsymbol{\varepsilon}(\boldsymbol{v}))_{\mathscr{H}}
$$

and the functional $j: V \times V \rightarrow \mathbb{R}$ by

$$
j(\boldsymbol{\eta}, \boldsymbol{v})=\int_{\Gamma_{3}} p_{\nu}\left(\eta_{\nu}-g\right) v_{\nu} d a+\int_{\Gamma_{3}} p_{\tau}\left(\eta_{\nu}-g\right)\left|\boldsymbol{v}_{\tau}\right| d a .
$$

Using the conditions (ii) and (4.20) it follows that for all $v \in V$ the functions $\boldsymbol{x} \mapsto p_{r}(\boldsymbol{x}, \boldsymbol{v}(\boldsymbol{x})-g(\boldsymbol{x}))(r=v, \tau)$ belong to $L^{2}\left(\Gamma_{3}\right)$ and therefore the integrals in (4.22) are well defined.

Let $f:[0, T] \rightarrow V$ by given by

$$
(\boldsymbol{f}(t), \boldsymbol{v})_{V}=\int_{\Omega} \boldsymbol{f}_{0}(t) \cdot \boldsymbol{v} d x+\int_{\Gamma_{2}} \boldsymbol{f}_{2}(t) \cdot \boldsymbol{v} d a \quad \forall \boldsymbol{v} \in V, t \in[0, T] .
$$

We note that conditions (4.19) imply

$$
f \in W^{1, \infty}(0, T ; V) .
$$

Finally we assume that the initial data satisfies

$$
\begin{gathered}
\boldsymbol{u}_{0} \in V, \\
a\left(\boldsymbol{u}_{0}, \boldsymbol{v}\right)+j\left(\boldsymbol{u}_{0}, \boldsymbol{v}\right) \geq(\boldsymbol{f}(0), \boldsymbol{v})_{V} \quad \forall \boldsymbol{v} \in V .
\end{gathered}
$$

It is straightforward to show that if $\{\boldsymbol{u}, \boldsymbol{\sigma}\}$ are sufficiently smooth functions satisfying (4.2), (4.3), (4.4), (4.5), and (4.6), then $\boldsymbol{u}(t) \in V$ and

$$
(\boldsymbol{\sigma}(t), \boldsymbol{\varepsilon}(\boldsymbol{v})-\boldsymbol{\varepsilon}(\dot{\boldsymbol{u}}(t)))_{\mathscr{H}}+j(\boldsymbol{u}(t), \boldsymbol{v})-j(\boldsymbol{u}(t), \dot{\boldsymbol{u}}(t)) \geq(\boldsymbol{f}(t), \boldsymbol{v}-\dot{\boldsymbol{u}}(t))_{V} \quad \forall \boldsymbol{v} \in V,
$$

for all $t \in[0, T]$. Therefore, using (4.1), (4.21), and (4.7) yields to the following variational formulation of Problem 1.

Problem 2. Find a displacement field $\boldsymbol{u}:[0, T] \rightarrow V$ such that

$$
\begin{gathered}
a(\boldsymbol{u}(t), \boldsymbol{v}-\dot{\boldsymbol{u}}(t))+ \\
\quad j(\boldsymbol{u}(t), \boldsymbol{v})-j(\boldsymbol{u}(t), \dot{\boldsymbol{u}}(t)) \\
\geq(\boldsymbol{f}(t), \boldsymbol{v}-\dot{\boldsymbol{u}}(t))_{V} \quad \forall \boldsymbol{v} \in V, \text { a.e. } t \in(0, T), \\
\boldsymbol{u}(0)=\boldsymbol{u}_{0} .
\end{gathered}
$$

Our main result, which we establish in the next section is the following. 
Theorem 4.1. Assume that conditions (i), (ii), (4.19), (4.20), (4.25), and (4.26) hold. Then there exists $L_{0}>0$ depending only on $\Omega, \Gamma_{1}, \Gamma_{3}$ and $\mathscr{E}$ such that if $L_{v}+$ $L_{\tau}<L_{0}$, then Problem 2 has at least a solution. Moreover, the solution satisfies $u \in W^{1, \infty}(0, T ; V)$.

Let now $\boldsymbol{u} \in W^{1, \infty}(0, T ; V)$ be the solution of Problem 2 and let $\boldsymbol{\sigma}$ be the stress field given by (4.1). Using (4.28) and (4.19) it can be shown that $\operatorname{Div} \sigma \in W^{1, \infty}(0, T ; H)$ and therefore $\sigma \in W^{1, \infty}\left(0, T ; \mathscr{H}_{1}\right)$. A pair of functions $\{\boldsymbol{u}, \boldsymbol{\sigma}\}$ which satisfies (4.1), (4.28), and (4.29) is called a weak solution of the problem (4.1), (4.2), (4.3), (4.4), (4.5), (4.6), and (4.7). We conclude that problem (4.1), (4.2), (4.3), (4.4), (4.5), (4.6), and (4.7) has at least a weak solution provided $L_{v}+L_{\tau}$ is sufficiently small. The critical value $L_{0}$ depends only on the elasticity operator and on the geometry of the problem but does not depend on the external forces, nor on the initial displacement.

The verification of the condition $L_{v}+L_{\tau}<L_{0}$ which guarantees the solvability of Problem 2 as well as its physical interpretation depends on the specific mechanical problem. For example, consider the mechanical Problem 1 in which the function $p_{v}$ is given by (4.8) and the function $p_{\tau}$ is given by (4.9) or by (4.10). It follows that assumption (ii)(a) is satisfied with $L_{v}=c_{v}$ and $L_{\tau}=\mu c_{v}$ and therefore the condition $L_{v}+L_{\tau}<L_{0}$ holds if $c_{v}(1+\mu)<L_{0}$, which may be interpreted as a smallness assumption involving the coefficients $c_{\nu}$ and $\mu$.

The important question of uniqueness of the solution to Problem 2 is left open. This is so even for the local elastic problem with normal compliance treated in [2], when the coefficient of friction and the load are assumed to be sufficiently small, as well as for the global elastic problem with normal compliance and friction studied in [3]. We finally remark that in the case of viscoelastic materials the unique solvability of quasistatic problems with normal compliance and friction may be proved without any smallness assumption on the data, see, for example, [15].

We end this section with an additional comment on the assumptions made on the contact functions $p_{v}$ and $p_{\tau}$. We remark that the choice of condition (ii) in Theorem 4.1 was made for simplicity since, as it will be shown in the next section, in this case it is easy to verify that the functional $j$ given by (4.22) satisfies the assumptions $(\mathrm{j} 1)$ (j6). However, the assumptions $(\mathrm{j} 1)-(\mathrm{j} 6)$ are quite general and may be verified in many other cases, even when the Lipschitz assumptions on the functions $p_{v}$ and $p_{\tau}$ are replaced by weaker assumptions. Considering different assumptions on the normal compliance function $p_{v}$ and on the friction bound function $p_{\tau}$ leads to different versions of Theorem 4.1 which may be proved using the abstract result provided by Theorem 2.1.

\section{Proof of Theorem 4.1}

The proof of Theorem 4.1 will be carried out in several steps and it is based on Theorem 2.1. We assume in what follows that (i), (ii), (4.19), (4.20), (4.25), and (4.26) hold and we start by investigating the properties of the functional $j$ given by (4.22). We remark that $j$ satisfies the condition (2). Moreover, we have the following results.

Lemma 5.1. The functional $j$ satisfies the assumptions ( $j 1)$ and (j2). 
Proof. Let $\boldsymbol{\eta}, \boldsymbol{u}, \overline{\boldsymbol{u}} \in V$ and let $\lambda \in] 0,1]$. Using (4.22) it results that

$$
\begin{aligned}
j(\boldsymbol{\eta}, \boldsymbol{u}-\overline{\boldsymbol{u}}-\lambda \boldsymbol{u})-j(\boldsymbol{\eta}, \boldsymbol{u}-\overline{\boldsymbol{u}}) \leq & -\lambda \int_{\Gamma_{3}} p_{v}\left(\eta_{v}-g\right) u_{v} d a \\
& -\lambda \int_{\Gamma_{3}} p_{\tau}\left(\eta_{v}-g\right)\left|\boldsymbol{u}_{\tau}-\overline{\boldsymbol{u}}_{\tau}\right| d a \\
& +\lambda \int_{\Gamma_{3}} p_{\tau}\left(\eta_{v}-g\right)\left|\overline{\boldsymbol{u}}_{\tau}\right| d a
\end{aligned}
$$

and, since $p_{\tau} \geq 0$ a.e. on $\Gamma_{3}$, we deduce

$$
j(\boldsymbol{\eta}, \boldsymbol{u}-\overline{\boldsymbol{u}}-\lambda \boldsymbol{u})-j(\boldsymbol{\eta}, \boldsymbol{u}-\overline{\boldsymbol{u}}) \leq-\lambda \int_{\Gamma_{3}} p_{\nu}\left(\eta_{v}-g\right) u_{\nu} d a+\lambda \int_{\Gamma_{3}} p_{\tau}\left(\eta_{v}-g\right)\left|\overline{\boldsymbol{u}}_{\tau}\right| d a .
$$

Therefore, by (2.5) we obtain

$$
j_{2}^{\prime}(\boldsymbol{\eta}, \boldsymbol{u}-\overline{\boldsymbol{u}} ;-\boldsymbol{u}) \leq-\int_{\Gamma_{3}} p_{\nu}\left(\eta_{\nu}-g\right) u_{\nu} d a+\int_{\Gamma_{3}} p_{\tau}\left(\eta_{\nu}-g\right)\left|\overline{\boldsymbol{u}}_{\tau}\right| d a \quad \forall \boldsymbol{\eta}, \boldsymbol{u}, \overline{\boldsymbol{u}} \in V .
$$

Now, we consider the sequences $\left\{\boldsymbol{u}_{n}\right\} \subset V,\left\{t_{n}\right\} \subset[0,1]$ and let $\overline{\boldsymbol{u}} \in V$. Using (ii) it follows that $p_{v}\left(t_{n} u_{n v}-g\right)\left(u_{n v}-g\right) \geq 0$ a.e. on $\Gamma_{3}$ and therefore (5.3) yields

$$
\begin{aligned}
& j_{2}^{\prime}\left(t_{n} \boldsymbol{u}_{n}, \boldsymbol{u}_{n}-\overline{\boldsymbol{u}} ;-\boldsymbol{u}_{n}\right) \\
& \quad \leq-\int_{\Gamma_{3}} g p_{v}\left(t_{n} u_{n v}-g\right) d a+\int_{\Gamma_{3}} p_{\tau}\left(t_{n} u_{n v}-g\right)\left|\overline{\boldsymbol{u}}_{\tau}\right| d a, \quad \forall n \in \mathbb{N} .
\end{aligned}
$$

Thus, since $g \geq 0, p_{v} \geq 0$ a.e. on $\Gamma_{3}$, using (ii) and (4.18), from the previous inequality we deduce

$$
\begin{aligned}
j_{2}^{\prime}\left(t_{n} \boldsymbol{u}_{n}, \boldsymbol{u}_{n}-\overline{\boldsymbol{u}} ;-\boldsymbol{u}_{n}\right) & \leq \int_{\Gamma_{3}} p_{\tau}\left(t_{n} u_{n v}-g\right)\left|\overline{\boldsymbol{u}}_{\tau}\right| d a \\
& \leq L_{\tau} \int_{\Gamma_{3}}\left(\left|u_{n v}\right|+|g|\right)\left|\overline{\boldsymbol{u}}_{\tau}\right| d a \\
& \leq L_{\tau} c_{B}\left(c_{B}\left|\boldsymbol{u}_{n}\right|_{V}+|g|_{L^{2}\left(\Gamma_{3}\right)}\right)|\overline{\boldsymbol{u}}|_{V} .
\end{aligned}
$$

It follows from the previous inequality that if $\left|\boldsymbol{u}_{n}\right|_{V} \rightarrow \infty$ then

$$
\liminf _{n \rightarrow \infty}\left[\frac{1}{\left|\boldsymbol{u}_{n}\right|_{V}^{2}} j_{2}^{\prime}\left(t_{n} \boldsymbol{u}_{n}, \boldsymbol{u}_{n}-\overline{\boldsymbol{u}} ;-\boldsymbol{u}_{n}\right)\right] \leq 0
$$

and we conclude that $j$ satisfies the assumption $(\mathrm{j} 1)$.

Now, we consider the sequences $\left\{\boldsymbol{u}_{n}\right\} \subset V,\left\{\boldsymbol{\eta}_{n}\right\} \subset V$ such that

$$
\begin{gathered}
\left|\boldsymbol{u}_{n}\right|_{V} \longrightarrow \infty, \\
\left|\boldsymbol{\eta}_{n}\right|_{V} \leq C \quad \forall n \in \mathbb{N},
\end{gathered}
$$


274 Evolutionary variational inequalities arising in quasistatic ...

where $C>0$. Let $\overline{\boldsymbol{u}} \in V$. Using (5.3) and (ii) we obtain

$$
\begin{aligned}
j_{2}^{\prime}\left(\boldsymbol{\eta}_{n}, \boldsymbol{u}_{n}-\overline{\boldsymbol{u}} ;-\boldsymbol{u}_{n}\right) \leq & \int_{\Gamma_{3}} p_{v}\left(\eta_{n v}-g\right)\left|u_{n v}\right| d a+\int_{\Gamma_{3}} p_{\tau}\left(\eta_{n v}-g\right)\left|\overline{\boldsymbol{u}}_{\tau}\right| d a \\
\leq & L_{v}\left(\left|\boldsymbol{\eta}_{n}\right|_{L^{2}\left(\Gamma_{3}\right)^{d}}+|g|_{L^{2}\left(\Gamma_{3}\right)}\right)\left|\boldsymbol{u}_{n}\right|_{L^{2}\left(\Gamma_{3}\right)^{d}} \\
& +L_{\tau}\left(\left|\boldsymbol{\eta}_{n}\right|_{L^{2}\left(\Gamma_{3}\right)^{d}}+|g|_{L^{2}\left(\Gamma_{3}\right)}\right)|\overline{\boldsymbol{u}}|_{L^{2}\left(\Gamma_{3}\right)^{d}} \quad \forall n \in \mathbb{N} .
\end{aligned}
$$

Using now (4.18) and (5.8) in the previous inequality yields

$$
j_{2}^{\prime}\left(\boldsymbol{\eta}_{n}, \boldsymbol{u}_{n}-\overline{\boldsymbol{u}} ;-\boldsymbol{u}_{n}\right) \leq c_{B}\left(C c_{B}+|g|_{L^{2}\left(\Gamma_{3}\right)}\right)\left(L_{\nu}\left|\boldsymbol{u}_{n}\right|_{V}+L_{\tau}|\overline{\boldsymbol{u}}|_{V}\right) \quad \forall n \in \mathbb{N} .
$$

Thus, from (5.10) and (5.7) we deduce that $j$ satisfies the assumption ( $\mathrm{j} 2)$.

Lemma 5.2. The functional $j$ satisfies the assumptions ( $j 3)$ and (j6).

Proof. Let $\left\{\boldsymbol{u}_{n}\right\} \subset V,\left\{\boldsymbol{\eta}_{n}\right\} \subset V$ be two sequences such that $\boldsymbol{\eta}_{n} \rightarrow \boldsymbol{\eta} \in V$ and $\boldsymbol{u}_{n} \rightarrow \boldsymbol{u} \in$ $V$. Using the compactness property of the trace map and (ii) it follows that

$$
\begin{gathered}
p_{r}\left(\eta_{n v}-g\right) \longrightarrow p_{r}\left(\eta_{v}-g\right) \quad \text { in } L^{2}\left(\Gamma_{3}\right) \quad(r=v, \tau), \\
\boldsymbol{u}_{n} \longrightarrow \boldsymbol{u} \text { in } L^{2}\left(\Gamma_{3}\right) .
\end{gathered}
$$

Therefore, we deduce from (5.11) and (5.12) that

$$
j\left(\boldsymbol{\eta}_{n}, \boldsymbol{v}\right) \longrightarrow j(\boldsymbol{\eta}, \boldsymbol{v}) \quad \forall \boldsymbol{v} \in V, \quad j\left(\boldsymbol{\eta}_{n}, \boldsymbol{u}_{n}\right) \longrightarrow j(\boldsymbol{\eta}, \boldsymbol{u}),
$$

which shows that the functional $j$ satisfies the condition $(\mathrm{j} 3)$.

Now, let $\left\{\boldsymbol{u}_{n}\right\}$ be a bounded subsequence of $V$, that is,

$$
\left|\boldsymbol{u}_{n}\right|_{V} \leq C \quad \forall n \in \mathbb{N}
$$

where $C>0$. We have

$$
\begin{aligned}
\left|j\left(\boldsymbol{\eta}_{n}, \boldsymbol{u}_{n}\right)-j\left(\boldsymbol{\eta}, \boldsymbol{u}_{n}\right)\right| \leq & \int_{\Gamma_{3}}\left|p_{v}\left(\eta_{n v}-g\right)-p_{v}\left(\eta_{v}-g\right)\right|\left|u_{n v}\right| d a \\
& +\int_{\Gamma_{3}}\left|p_{\tau}\left(\eta_{n v}-g\right)-p_{\tau}\left(\eta_{v}-g\right)\right|\left|\boldsymbol{u}_{n \tau}\right| d a
\end{aligned}
$$

and, using again (4.18), we deduce

$$
\begin{aligned}
\left|j\left(\boldsymbol{\eta}_{n}, \boldsymbol{u}_{n}\right)-j\left(\boldsymbol{\eta}, \boldsymbol{u}_{n}\right)\right| \leq c_{B}( & \left|p_{\nu}\left(\eta_{n v}-g\right)-p_{\nu}\left(\eta_{v}-g\right)\right|_{L^{2}\left(\Gamma_{3}\right)} \\
& \left.+\left|p_{\tau}\left(\eta_{n v}-g\right)-p_{\tau}\left(\eta_{v}-g\right)\right|_{L^{2}\left(\Gamma_{3}\right)}\right)\left|\boldsymbol{u}_{n}\right|_{V} .
\end{aligned}
$$

It follows now from (5.11), (5.14), and (5.16) that $j$ satisfies assumption ( $\mathrm{j} 6)$.

Lemma 5.3. The functional $j$ satisfies the assumption (j5). Moreover,

$$
j(\boldsymbol{u}, \boldsymbol{v}-\boldsymbol{u})-j(\boldsymbol{v}, \boldsymbol{v}-\boldsymbol{u}) \leq c_{B}^{2}\left(L_{v}+L_{\tau}\right)|\boldsymbol{u}-\boldsymbol{v}|_{V}^{2} \quad \forall \boldsymbol{u}, \boldsymbol{v} \in V .
$$


Proof. Let $\boldsymbol{\eta}, \boldsymbol{u} \in V$. Using (4.22) and (ii) it follows that

$$
\begin{aligned}
|j(\boldsymbol{\eta}, \boldsymbol{u})| & \leq \int_{\Gamma_{3}} p_{\nu}\left(\eta_{\nu}-g\right)\left|u_{\nu}\right| d a+\int_{\Gamma_{3}} p_{\tau}\left(\eta_{\nu}-g\right)\left|\boldsymbol{u}_{\tau}\right| d a \\
& \leq L_{\nu}\left|\eta_{\nu}-g\right|_{L^{2}\left(\Gamma_{3}\right)}\left|u_{\nu}\right|_{L^{2}\left(\Gamma_{3}\right)}+L_{\tau}\left|\eta_{\nu}-g\right|_{L^{2}\left(\Gamma_{3}\right)}\left|\boldsymbol{u}_{\tau}\right|_{L^{2}\left(\Gamma_{3}\right)^{d}}
\end{aligned}
$$

and, keeping in mind (4.18), we find

$$
|j(\boldsymbol{\eta}, \boldsymbol{u})| \leq c_{B}\left(L_{v}+L_{\tau}\right)\left(c_{B}|\boldsymbol{\eta}|_{V}+|g|_{L^{2}\left(\Gamma_{3}\right)}\right)|\boldsymbol{u}|_{V}
$$

which implies (j5).

Now, let $\boldsymbol{u}, \boldsymbol{v} \in V$. Using again (4.22) and (ii) it follows that

$$
\begin{aligned}
j(\boldsymbol{u}, \boldsymbol{v}-\boldsymbol{u})-j(\boldsymbol{v}, \boldsymbol{v}-\boldsymbol{u})= & \int_{\Gamma_{3}}\left(p_{\nu}\left(u_{v}-g\right)-p_{v}\left(v_{v}-g\right)\right)\left(v_{v}-u_{v}\right) d a \\
& +\int_{\Gamma_{3}}\left(p_{\tau}\left(u_{v}-g\right)-p_{\tau}\left(v_{v}-g\right)\right)\left(\left|\boldsymbol{v}_{\tau}-\boldsymbol{u}_{\tau}\right|\right) d a \\
\leq & L_{v} \int_{\Gamma_{3}}\left|u_{v}-v_{v}\right|^{2} d a+L_{\tau} \int_{\Gamma_{3}}\left|u_{v}-v_{v}\right|\left|\boldsymbol{v}_{\tau}-\boldsymbol{u}_{\tau}\right| d a \\
\leq & \left(L_{v}+L_{\tau}\right) \int_{\Gamma_{3}}|\boldsymbol{u}-\boldsymbol{v}|^{2} d a .
\end{aligned}
$$

Using now (4.18) in the previous inequality we deduce (5.17).

We have all the ingredients to prove the theorem.

Proof of Theorem 4.1. Using the conditions (i) and (4.17) we see that the bilinear form $a$ defined by (4.21) is symmetric, continuous and coercive, that is,

$$
a(\boldsymbol{v}, \boldsymbol{v}) \geq m|\boldsymbol{v}|_{V}^{2} \quad \forall \boldsymbol{v} \in V .
$$

Let $L_{0}=m / c_{B}^{2}$. Clearly $L_{0}$ depends only on $\Omega, \Gamma_{1}, \Gamma_{3}$, and $\mathscr{E}$. Let now assume that $L_{v}+L_{\tau}<L_{0}$. Then, there exists $c_{0} \in \mathbb{R}$ such that $c_{B}^{2}\left(L_{v}+L_{\tau}\right) \leq c_{0}<m$. Using (5.17) we obtain

$$
j(\boldsymbol{u}, \boldsymbol{v}-\boldsymbol{u})-j(\boldsymbol{v}, \boldsymbol{v}-\boldsymbol{u}) \leq c_{0}|\boldsymbol{u}-\boldsymbol{v}|_{V}^{2} \quad \forall \boldsymbol{u}, \boldsymbol{v} \in V
$$

and we conclude that the functional $j$ satisfies the condition ( $\mathrm{j} 4)$. Using Lemmas 5.1, 5.2, 5.3, (4.24), (4.25), (4.26), and Theorem 2.1, we deduce that Problem 2 has at least a solution $\boldsymbol{u} \in W^{1, \infty}(0, T ; V)$.

\section{Other quasistatic frictional contact problems}

In this section, we consider two quasistatic frictional contact problems for linear elastic materials which may be set in the variational formulation (2.14). We use Corollary 2.2 to prove the existence, the uniqueness and the Lipschitz continuous dependence of 
the weak solution with respect to the data. The results we present here extend to the quasistatic case some results obtained in $[6,13]$ where the corresponding mechanical problems were considered in the static case. The physical setting is similar to that in Section 4 but the contact conditions on $\Gamma_{3} \times(0, T)$ are different. Everywhere in what follows we use the same notation for the spaces $H, H_{1}, \mathscr{H}$, and $\mathscr{H}_{1}$. We assume that the elasticity operator $\mathscr{E}$ satisfies (i) and that the body forces and tractions satisfy (4.19). We also use the notation (4.21).

6.1. Bilateral contact with Tresca's friction law. In the first example we consider a bilateral contact modeled by Tresca's friction law (cf. $[1,6])$, that is,

$$
\left.\begin{array}{l}
u_{v}=0, \quad\left|\boldsymbol{\sigma}_{\tau}\right| \leq g, \\
\left|\boldsymbol{\sigma}_{\tau}\right|<g \Longrightarrow \dot{\boldsymbol{u}}_{\tau}=\mathbf{0}, \\
\left|\boldsymbol{\sigma}_{\tau}\right|=g \Longrightarrow \boldsymbol{\sigma}_{\tau}=-\lambda \dot{\boldsymbol{u}}_{\tau}, \quad \lambda \geq 0
\end{array}\right\} \text { on } \Gamma_{3} \times(0, T) .
$$

Here $u_{v}$ represents the normal displacement, $\dot{\boldsymbol{u}}_{\tau}$ denotes the tangential velocity, $\boldsymbol{\sigma}_{\tau}$ is the tangential force on the contact boundary and $g$ is the friction bound, that is, the magnitude of the limiting friction traction at which slip begins. In (6.1) the strong inequality holds in the stick zone and the equality in the slip zone. The contact is assumed to be bilateral, that is, there is no loss of the contact during the process.

With this assumption, the mechanical problem of frictional contact we consider is the following.

Problem 3. Find a displacement $\boldsymbol{u}: \Omega \times[0, T] \rightarrow \mathbb{R}^{d}$ and a stress field $\boldsymbol{\sigma}: \Omega \times$ $[0, T] \rightarrow S_{d}$ which satisfy (4.1), (4.2), (4.3), (4.4), (4.7), and (6.1).

Let $V$ denote the closed subspace of $H_{1}$ given by

$$
V=\left\{\boldsymbol{v} \in H_{1} \mid \boldsymbol{v}=\mathbf{0} \text { on } \Gamma_{1}, v_{v}=0 \text { on } \Gamma_{3}\right\},
$$

endowed with the inner product (4.17).

We assume in what follows that the friction bound satisfies

$$
g \in L^{\infty}\left(\Gamma_{3}\right), \quad g \geq 0 \text { a.e. on } \Gamma_{3}
$$

and we define the friction functional $\varphi: V \rightarrow \mathbb{R}$ by

$$
\varphi(\boldsymbol{v})=\int_{\Gamma_{3}} g\left|\boldsymbol{v}_{\tau}\right| d a .
$$

Finally we assume that the initial data satisfies

$$
\boldsymbol{u}_{0} \in V, \quad a\left(\boldsymbol{u}_{0}, \boldsymbol{v}\right)+\varphi(\boldsymbol{v}) \geq(\boldsymbol{f}(0), \boldsymbol{v})_{V} \quad \forall \boldsymbol{v} \in V,
$$

where $f$ is given by (4.23).

Using the arguments of [1] we deduce the following variational formulation of the quasistatic Problem 3. 
Problem 4. Find a displacement field $\boldsymbol{u}:[0, T] \rightarrow V$ such that

$$
\begin{gathered}
a(\boldsymbol{u}(t), \boldsymbol{v}-\dot{\boldsymbol{u}}(t))+\varphi(\boldsymbol{v})-\varphi(\dot{\boldsymbol{u}}(t)) \geq(\boldsymbol{f}(t), \boldsymbol{v}-\dot{\boldsymbol{u}}(t))_{V} \quad \forall \boldsymbol{v} \in V, \text { a.e. } t \in(0, T), \\
\boldsymbol{u}(0)=\boldsymbol{u}_{0}
\end{gathered}
$$

In the study of Problem 4 we remark that assumption (6.3) implies that $\varphi$ is a continuous seminorm on $V$. Moreover, assumptions (i) and (4.19) imply that $a$ and $\boldsymbol{f}$ satisfy conditions (1) and (2.2), respectively. Therefore, using Corollary 2.2 we obtain the following result.

Theorem 6.1. Assume that conditions (i), (4.19), (6.3), and (6.5) hold. Then Problem 4 has a unique solution $u \in W^{1, \infty}(0, T ; V)$. Moreover, the mapping $\left(\boldsymbol{f}, \boldsymbol{u}_{0}\right) \mapsto \boldsymbol{u}$ is Lipschitz continuous from $W^{1, \infty}(0, T ; V) \times V$ to $L^{\infty}(0, T ; V)$.

Let now $\boldsymbol{u} \in W^{1, \infty}(0, T ; V)$ be the solution of the Problem 4 and let $\sigma$ be the stress field given by (4.1). As is Section 4 it can be shown that $\sigma \in W^{1, \infty}\left(0, T ; \mathscr{H}_{1}\right)$. A pair of functions $\{\boldsymbol{u}, \boldsymbol{\sigma}\}$ which satisfies (6.6), (6.7), and (4.1) is called a weak solution of the bilateral contact problem with Tresca's friction law (4.1), (4.2), (4.3), (4.4), (4.7), and (6.1). We conclude by Theorem 6.1 that the Problem 3 has a unique weak solution which depends Lipschitz continuously on the data.

6.2. Contact with simplified Coulomb's friction law. Consider a contact problem modeled by a simplified version of Coulomb's law of dry friction (cf. $[6,13])$, that is,

$$
\left.\begin{array}{l}
\sigma_{\nu}=S, \quad\left|\sigma_{\tau}\right| \leq \mu\left|\sigma_{\nu}\right| \\
\left|\sigma_{\tau}\right|<\mu\left|\sigma_{\nu}\right| \Longrightarrow \dot{\boldsymbol{u}}_{\tau}=\mathbf{0}, \\
\left|\boldsymbol{\sigma}_{\tau}\right|=\mu\left|\sigma_{\nu}\right| \Longrightarrow \boldsymbol{\sigma}_{\tau}=-\lambda \dot{\boldsymbol{u}}_{\tau}, \quad \lambda \geq 0
\end{array}\right\} \text { on } \Gamma_{3} \times(0, T)
$$

Here $\sigma_{v}$ denotes the normal stress on the contact boundary, $\sigma_{\tau}$ is the tangential force on the contact boundary, $\dot{\boldsymbol{u}}_{\tau}$ denotes the tangential velocity, $S \in L^{\infty}\left(\Gamma_{3}\right)$ is a given function and $\mu$ is the coefficient of friction.

With this assumption, the mechanical problem of frictional contact we consider is the following.

Problem 5. Find a displacement $\boldsymbol{u}: \Omega \times[0, T] \rightarrow \mathbb{R}^{d}$ and a stress field $\boldsymbol{\sigma}: \Omega \times$ $[0, T] \rightarrow S_{d}$ which satisfy (4.1), (4.2), (4.3), (4.4), (4.7), and (6.8).

Let $V$ denote the closed subspace of $H_{1}$ given by

$$
V=\left\{\boldsymbol{v} \in H_{1} \mid \boldsymbol{v}=\mathbf{0} \text { on } \Gamma_{1}\right\},
$$

endowed with the inner product (4.17).

We assume that the given normal stress satisfies

$$
S \in L^{\infty}\left(\Gamma_{3}\right)
$$


278 Evolutionary variational inequalities arising in quasistatic ...

and the coefficient of friction is such that

$$
\mu \in L^{\infty}\left(\Gamma_{3}\right), \quad \mu \geq 0 \text { a.e. on } \Gamma_{3} \text {. }
$$

We define the friction functional $\varphi: V \rightarrow \mathbb{R}$ by

$$
\varphi(\boldsymbol{v})=\int_{\Gamma_{3}} \mu|S|\left|\boldsymbol{v}_{\tau}\right| d a
$$

and let

$$
(\boldsymbol{f}(t), \boldsymbol{v})_{V}=\int_{\Omega} \boldsymbol{f}_{0}(t) \cdot \boldsymbol{v} d x+\int_{\Gamma_{2}} \boldsymbol{f}_{2}(t) \cdot \boldsymbol{v} d a+\int_{\Gamma_{3}} S v_{\nu} d a \quad \forall \boldsymbol{v} \in V, t \in[0, T]
$$

With these notation, the variational formulation of the mechanical Problem 5 is as follows.

Problem 6. Find a displacement field $\boldsymbol{u}:[0, T] \rightarrow V$ such that $u$ satisfies (6.6) and (6.7).

In the study of Problem 6 we remark that assumptions (6.10) and (6.11) imply that $\varphi$ is a continuous seminorm on $V$. Moreover, condition (i) implies (1) and conditions (4.19) and (6.10) imply the regularity $f \in W^{1, \infty}(0, T ; V)$. Therefore, using Corollary 2.2 we obtain the following result.

Theorem 6.2. Assume that conditions (i), (4.19), (6.5), (6.10), and (6.11) hold. Then Problem 6 has a unique solution $u \in W^{1, \infty}(0, T ; V)$. Moreover, the mapping $\left(\boldsymbol{f}, \boldsymbol{u}_{0}\right) \mapsto \boldsymbol{u}$ is Lipschitz continuous from $W^{1, \infty}(0, T ; V) \times V$ to $L^{\infty}(0, T ; V)$.

As in the previous example, we conclude by Theorem 6.2 that the mechanical Problem 6 has a unique weak solution which depends Lipschitz continuously on the data.

\section{References}

[1] A. Amassad and M. Sofonea, Analysis of a quasistatic viscoplastic problem involving Tresca friction law, Discrete Contin. Dynam. Systems 4 (1998), no. 1, 55-72. MR 98j:73042. Zbl 991.51622.

[2] L.-E. Andersson, A quasistatic frictional problem with normal compliance, Nonlinear Anal. 16 (1991), no. 4, 347-369. MR 91m:73066. Zbl 722.73061.

[3] A global existence result for a quasistatic contact problem with friction, Adv. Math. Sci. Appl. 5 (1995), no. 1, 249-286. MR 96b:35123. Zbl 832.35056.

[4] H. Brézis, Problemes unilateraux [Unilateral problems], J. Math. Pures Appl. (9) 51 (1972), 1-168 (French). MR 55\#1166. Zbl 237.35001.

[5] M. Cocu, E. Pratt, and M. Raous, Formulation and approximation of quasistatic frictional contact, Internat. J. Engrg. Sci. 34 (1996), no. 7, 783-798. MR 97g:73089. Zb1 900.73684.

[6] G. Duvaut and J.-L. Lions, Inequalities in Mechanics and Physics, Grundlehren der Mathematischen Wissenschaften, vol. 219, Springer-Verlag, Berlin, 1976, translated from the French by C. W. John. MR 58\#25191. Zbl 331.35002. 
[7] W. Han and M. Sofonea, Analysis and numerical approximation of an elastic frictional contact problem with normal compliance, Appl. Math. (Warsaw) 26 (1999), no. 4, 415435. CMP 1737179.

[8] N. Kikuchi and J. T. Oden, Contact Problems in Elasticity: a Study of Variational Inequalities and Finite Element Methods, SIAM Studies in Applied Mathematics, vol. 8, Society for Industrial and Applied Mathematics (SIAM), Philadelphia, 1988. MR 89j:73097. Zbl 685.73002.

[9] A. Klarbring, A. Mikelić, and M. Shillor, Frictional contact problems with normal compliance, Internat. J. Engrg. Sci. 26 (1988), no. 8, 811-832. MR 89j:73098. Zbl 662.73079.

[10] J. A. C. Martins and J. T. Oden, Existence and uniqueness results for dynamic contact problems with nonlinear normal and friction interface laws, Nonlinear Anal. 11 (1987), no. 3, 407-428. MR 88e:73025. Zbl 672.73079.

[11] D. Motreanu and M. Sofonea, Quasivariational inequalities and applications in frictional contact problems with normal compliance, Adv. Math. Sci. Appl. 10 (2000), no. 1, 103118. CMP 1769 178. Zbl 991.61412.

[12] J. Nečas and I. Hlaváček, Mathematical Theory of Elastic and Elasto-plastic Bodies: an Introduction, Studies in Applied Mechanics, vol. 3, Elsevier Scientific Publishing Co., Amsterdam, 1980. MR 82h:73002. Zbl 448.73009.

[13] P. D. Panagiotopoulos, Inequality Problems in Mechanics and Applications. Convex and nonconvex energy functions, Birkhäuser Boston, Boston, 1985. MR 88h:49003. Zbl 579.73014.

[14] M. Raous, M. Jean, and J. J. Moreau (eds.), Contact Mechanics, Plenum Press, New York, 1995.

[15] M. Rochdi, M. Shillor, and M. Sofonea, Quasistatic viscoelastic contact with normal compliance and friction, J. Elasticity 51 (1998), no. 2, 105-126. MR 99k:73110. Zbl 921.73231.

[16] M. Sofonea, On a contact problem for elastic-viscoplastic bodies, Nonlinear Anal. 29 (1997), no. 9, 1037-1050. MR 98e:73125. Zbl 918.73098.

[17] N. Strömberg, Continuum thermodynamics of contact, friction and wear, Ph.D. thesis, Linköping University, Sweden, 1995.

[18] N. Strömberg, L. Johansson, and A. Klarbring, Derivation and analysis of a generalized standard model for contact, friction and wear, Internat. J. Solids Structures 33 (1996), no. 13, 1817-1836. MR 97b:73085. Zbl 926.74012.

Dumitru Motreanu: Départment de Mathématiques, Université de Perpignan, 52 Avenue de Villeneuve, 66860 Perpignan, France

E-mail address: motreanu@univ-perp.fr

Mircea Sofonea: Laboratoire de Théorie des Systèmes, Université de Perpignan, 52 Avenue de Villeneuve, 66860 Perpignan, France

E-mail address: sofonea@univ-perp.fr 


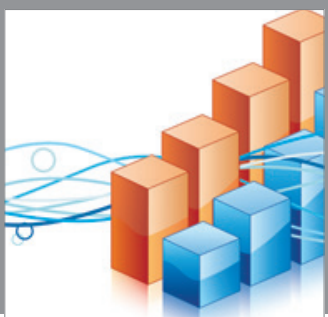

Advances in

Operations Research

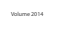

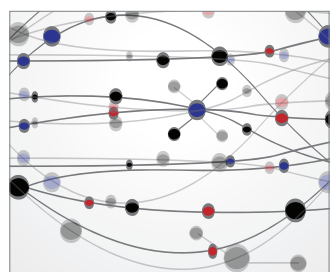

\section{The Scientific} World Journal
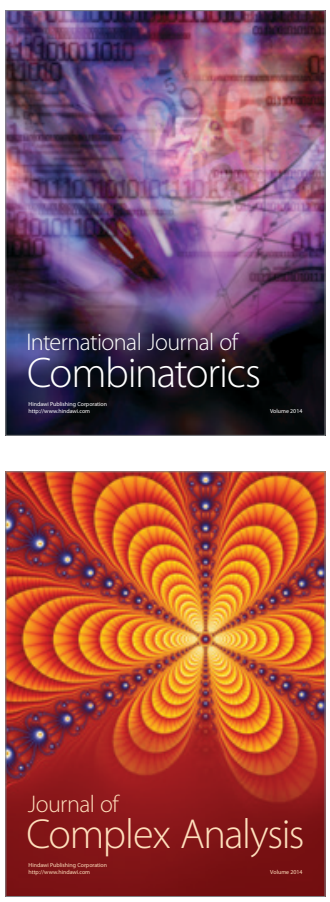

International Journal of

Mathematics and

Mathematical

Sciences
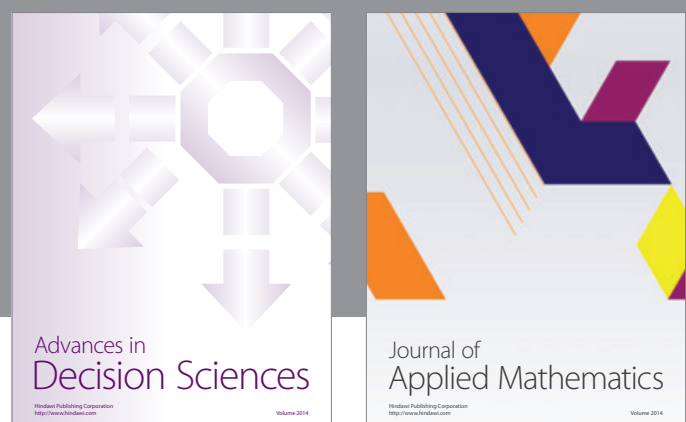

Journal of

Applied Mathematics
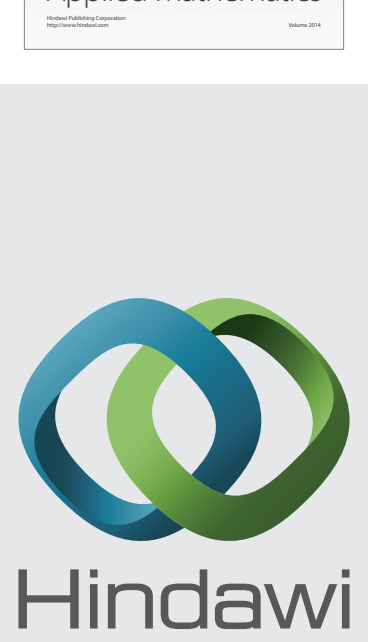

Submit your manuscripts at http://www.hindawi.com
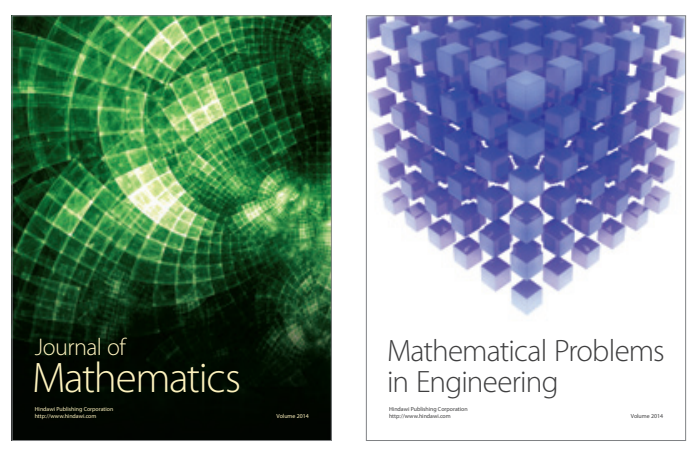

Mathematical Problems in Engineering
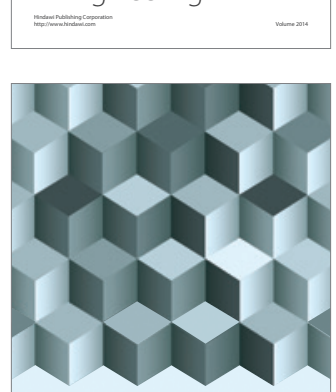

Journal of

Function Spaces
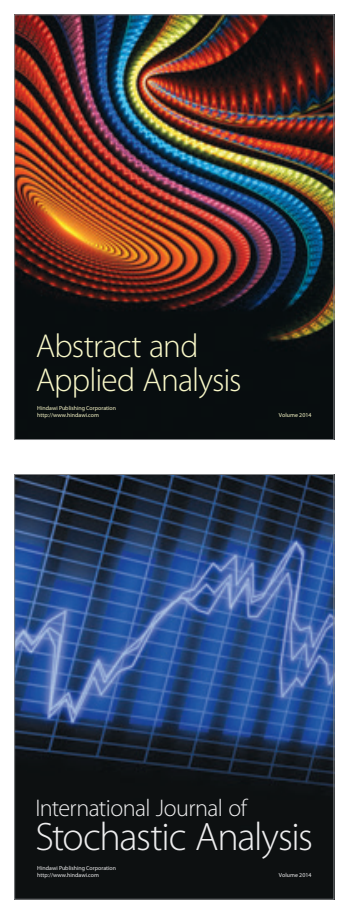

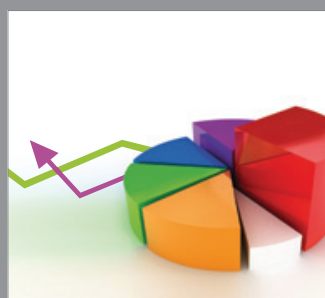

ournal of

Probability and Statistics

Promensencen
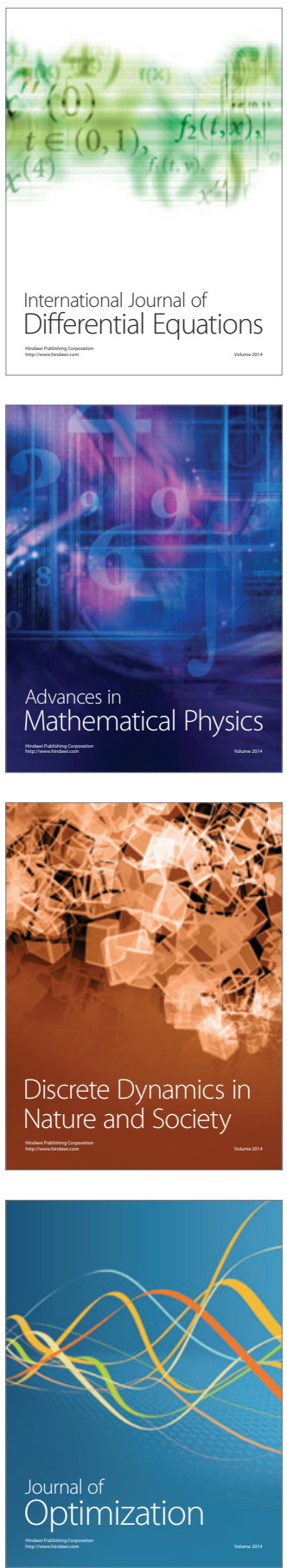